\title{
Artificial Noise Based Beamforming for the MISO VLC Wiretap Channel
}

\author{
Mohamed Amine Arfaoui, Hajar Zaid, Zouheir Rezki, Ali Ghrayeb, Anas Chaaban and Mohamed Slim Alouini
}

\begin{abstract}
This paper investigates the secrecy performance of the multiple-input single-output (MISO) visible light communication (VLC) wiretap channel. The considered system model comprises three nodes: a transmitter (Alice) equipped with multiple fixtures of LEDs, a legitimate receiver (Bob) and an eavesdropper (Eve), each equipped with one photo-diode (PD). The VLC channel is modeled as a real-valued amplitude-constrained Gaussian channel. Eve is assumed to be randomly located in the same area as Bob. Due to this, artificial noise (AN)-based beamforming is adopted as a transmission strategy in order to degrade Eve's signal-to-noise ratio (SNR). Assuming discrete input signaling, we derive an achievable secrecy rate in a closed-form expression as a function of the beamforming vectors and the input distribution. We investigate the average secrecy performance of the system using stochastic geometry to account for the location randomness of Eve. We also adopt the truncated discrete generalized normal (TDGN) as a discrete input distribution. We present several examples through which we confirm the accuracy of the analytical results via Monte Carlo simulations. The results also demonstrate that the TDGN distribution, albeit being not optimal, yields performance close to the secrecy capacity.
\end{abstract}

Keywords-Achievable secrecy rate, beamforming, MISO, stochastic geometry, TDGN, VLC.

\section{INTRODUCTION}

\section{A. Motivation}

Visible light communication (VLC) is a promising technology that has gained significant attention due to its high data rates and low cost of deployment. Compared to radio frequency (RF) communications, especially for indoor environments, VLC offers several advantages including robustness against interference and abundance of the available spectrum [1]. Various aspects of VLC systems have been studied in the literature. In [2], the authors studied point-to-point VLC links and proposed suitable modulation schemes. The authors in [3] studied the performance of VLC systems in terms of transmit

M.-A. Arfaoui is within the Concordia Institute for Information Systems Engineering (CIISE), Concordia University, Montreal, QC H3G 1M8, Canada. Email: m_arfaou@encs.concordia.ca.

H. Zaid is with the Electronics Computer and Telecommunications (ECT) department, National School of Applied Sciences (NSAC), Oujda, Maroc. Email: Zaidehajar@gmail.com.

Z. Rezki is with the Electrical and Computer Engineering Department, University of Idaho, Moscow, ID 83844 USA. Email: zrezki@uidaho.edu.

A. Ghrayeb is within the Electrical and Computer Engineering Department, Texas A\&M University at Qatar. Email: ali.ghrayeb@qatar.tamu.edu.

A. Chaaban is with the School of Engineering, The University of British Columbia, Kelowna, BC V1V 1V7, Canada. Email: anas.chaaban@ubc.ca.

M.-S. Alouini is with the Division of Computer, Electrical, and Mathematical Sciences and Engineering, King Abdullah University of Science and Technology, Thuwal 23955-6900, Saudi Arabia. Email: slim.alouini@kaust.edu.sa.

This work was supported by Qatar National Research Fund (a member of Qatar Foundation) under NPRP Grant NPRP8-052-2-029. The statements made herein are solely the responsibility of the authors. data rates, channel bandwidth and signal-to-interference-plusnoise (SINR) ratio. In [4]-[6], the authors presented a review of VLC, whereas the authors of [7] discussed its potential for indoor communications. In [8]-[10], the authors studied fundamental limits of optical wireless channels. In [11], the viability of VLC for $5 \mathrm{G}$ wireless networks was investigated.

Although VLC systems are less susceptible to eavesdropping than RF systems since light does not penetrate through walls, they become as vulnerable as their RF counterparts when their nodes are deployed in public areas and/or when there are large windows [12]. Thus, security for VLC systems is as important as it is for RF systems. Secrecy in wireless communication systems may be enhanced by introducing physical layer security (PLS) techniques [13]. In fact, PLS has been applied to a wide range of RF applications in an effort to improve the overall security by complementing existing cryptography-based security techniques. The potential of PLS stems from its ability of leveraging features of the surrounding environments via sophisticated encoding techniques at the physical layer [14], [15]. Indeed, PLS schemes can be applied in the same spirit to VLC systems.

There exist many specificities that characterize VLC systems, leading to major differences compared to RF systems. Precisely, VLC channels are quasi-static and real valued channels, which seemingly simplify the application of PLS techniques. However, due to the limited dynamic range of the LEDs [16], VLC systems impose a peak-power constraint, i.e., amplitude constraint, on the channel input, which makes unbounded inputs not admissible. As a result, the performance and the optimization of PLS schemes must be revised in the VLC context due to its different operating constraints.

\section{B. Related Work}

The secrecy performance for the MISO RF wiretap channel was widely investigated in the literature. In [17]-[20], the problem was investigated under perfect eavesdropper's channel state information (CSI) and average power constraint. In [17], [18], it was shown that Gaussian signaling, along with beamforming, is the optimal transmission strategy, and closed-form secrecy capacity expressions were derived. The same problem but with imperfect eavesdropper's CSI was considered in [21][24], where robust beamforming and worst-case secrecy rate maximization were investigated in [21], [22] and artificial noise schemes where proposed in [23], [24].

From an information-theoretic point of view and similar to the average power constrained case, one can use the existing single-letter description for the rate-equivocation region of 
the Gaussian wiretap channel under peak-power constraint as in [25]. However, unlike the average power constrained case, the corresponding optimization problems are harder to solve explicitly under peak-power constraints, which makes the derivation of the secrecy capacity for this class of wiretap channels becomes more complex. The authors in [26] showed that the secrecy capacity achieving distribution of the MISO wiretap channel under peak-power constraint is discrete with a finite support set. However, neither the secrecy capacity nor its achieving distribution were determined in closed-form and they can be only found via numerical methods. Due to this and since VLC channels fall within this category, the majority of works relative to securing VLC systems consider only continuous input signaling.

Although the adoption of PLS techniques developed for RF channels for VLC channels may not be straightforward since RF signals are complex-valued, which is fundamentally different from the real and bounded VLC signals, the problem of secure MISO VLC systems was investigated in [27]-[35]. ${ }^{1}$ Under perfect eavesdropper's CSI, beamforming [27]-[29] and artificial noise [30]-[35] are among the schemes employed in PLS that aim at enhancing the secrecy performance of VLC systems. The results of [27]-[35] are based on the assumption that the location of the eavesdropper is either exactly known to the transmitter or confined within a known bounded area, where only continuous input distributions have been employed.

The assumption that the eavesdropper's CSI is perfectly known to Alice is justifiable only in some scenarios, e.g., the eavesdropper is an authorized user in the network but confidential messages shall be exchanged between the transmitter and a legitimate receiver. However, such assumption is not valid if the eavesdropper is passive or a malicious user. In this case, one may assume that the eavesdropper is randomly located within the area of interest [36]-[39]. This scenario was considered for MISO VLC wiretap channels in [39], [40], where the authors employed beamforming in conjunction of continuous input signaling and derived closedform expressions for the average achievable secrecy rate. However, adopting continuous input signaling is unrealistic since digital data streams should be transmitted. In addition, discrete input signaling has been shown to be optimal for MISO VLC channels [26]. To the best of our knowledge, the use of artificial noise schemes in conjunction of discrete input for securing MISO VLC wiretap channels was not investigated in the literature, which is the focus of this paper.

\section{Contributions}

In this paper, we considered a MISO VLC wiretap channel comprising a transmitter (Alice), a legitimate receiver (Bob) and a randomly located eavesdropper (Eve). Alice is equipped with $N$ fixtures of LEDs, whereas Bob and Eve are each equipped with a single photo-diode (PD). The transmitted signal is subject to a peak-power constraint. The objective of the paper is enhancing the secrecy performance of the

\footnotetext{
${ }^{1}$ While the papers [29]-[31] tackle the same problem considered here, this paper presents new contributions including the discreetness of the input signaling and the randomness of the eavesdropper's location.
}

communication link between Alice and Bob using an artificial noise (AN) based beamforming scheme. The contributions of this paper are summarized as follows.

- We derive a closed-form expression for an achievable secrecy rate as a function of the precoding vectors for the information-bearing signal and the AN signal, and for any discrete input distribution.

- We use some approximations to obtain an analytical solution to the derived secrecy rate expression. To this end, we first derive upper and lower bounds on the obtained achievable secrecy rate and we maximize these bounds using the convex-concave procedure (CCP). The obtained solutions are then injected into the original average achievable secrecy rate, which leads to a suboptimal secrecy rate.

- We analyze the complexity of the proposed (suboptimal) scheme and show that it offers a significant reduction in complexity compared to that of the brute force methods with little degradation in performance.

- In the numerical examples, we adopt the truncated discrete generalized normal (TDGN) distribution for the information and AN signals and optimize over its parameters to maximize the achievable secrecy rate. We demonstrate that, although it is not optimal, the TDGN yields performance close that of the capacity limit.

\section{Outline and Notations}

The rest of the paper is organized as follows. Section II presents the system model. Section III presents the secrecy performance with perfect Eve's CSI. Section IV presents secrecy performance with randomly located Eve. Sections V and VI represent simulation results and conclusions, respectively.

The following notation is adopted throughout the paper. Upper case bold characters denote matrices and lower case bold characters denote column vectors. The set of natural numbers is denoted by $\mathbb{N}$. The set of $N$-dimensional real-valued numbers is denoted by $\mathbb{R}^{N}$ and the set of $N$-dimensional non-negative real-valued numbers is denoted by $\mathbb{R}_{+}^{N}$. $\mathbb{N}^{*}$ denotes the set $\mathbb{N} \backslash\{0\}$. Matrix transposition is denoted by the superscript $\{\cdot\}^{T} \cdot\|\cdot\|_{p}$, for $p=1,2, \ldots, \infty$, denotes the p-norm. $\mathcal{N}\left(0, \sigma^{2}\right)$ denotes the Gaussian probability distribution with zero-mean and $\sigma^{2}$ variance. For a random scalar variable $\mathbf{s}, p_{s}$ denotes its probability density/mass function (pdf/pmf), whether $s$ is continuous and discrete, respectively. The expected value is denoted by $\mathbb{E}(\cdot)$, the differential entropy is denoted by $h(\cdot)$ and the mutual information by $I(\cdot ; \cdot)$. Superscript $[C]^{+}$denotes $\max (C, 0)$. We use $\log (\cdot)$, without a base, to denote natural logarithms and information rates are specified in (Nats/s/Hz). Subscripts $\{\cdot\}_{B}$ and $\{\cdot\}_{E}$ denote Bob's and Eve's relevance, respectively.

\section{SySTEM MODEL}

\section{A. The VLC Channel Model}

We consider a DC-biased intensity-modulation directdetection (IM-DD) scheme where the transmit element is an illumination LED driven by a fixed bias $I_{D C} \in \mathbb{R}_{+}$. The DC-offset sets the average radiated optical power and, 
consequently, settles the illumination level. The data signal $s \in \mathbb{R}$ is a zero-mean current signal superimposed on $I_{D C}$ to modulate the instantaneous optical power emitted from the LED. In order to maintain linear current-light conversion and avoid clipping distortion, the total current $I_{D C}+s$ must be constrained within some range $I_{D C} \pm \nu I_{D C}$ where $\nu \in[0,1]$ is the modulation index [27]. Consequently, $s$ must satisfy a peak-power constraint expressed as $|s| \leqslant A$, where $A=\nu I_{D C}$. After that, the total current $I_{D C}+s$ is converted into an optical power $P_{T}=\eta\left(I_{D C}+s\right)$ and transmitted by the LED, where $\eta$ denotes its conversion factor. At the receiver side, the receiver's PD, with a responsivity $R_{p}$, converts the incident optical power into a proportional current. Finally the DC-offset $I_{D C}$ is removed and a transimpedance amplifier, with gain $T$, is used to produce a voltage signal $y \in \mathbb{R}$, which is a scaled, but noisy, version of the transmitted signal $s$.

Armed with the above description, the received signal is expressed as

$$
y=h s+n,
$$

where $y$ represents the received signal, $s$ represents the zeromean transmitted signal subject to the amplitude constraint $|s| \leq A$, such that $A=\nu I_{D C}, n$ represents the additive white Gaussian noise (AWGN) and $h=\eta R T g \in \mathbb{R}_{+}$represents the channel gain, in which $g$ denotes the path gain of the optical link. Assuming that the considered LED has a Lambertian emission pattern, the path gain is expressed as [41], [42]

$$
g= \begin{cases}\frac{1}{2 \pi}(m+1) \cos ^{m}(\theta) \frac{A_{R X}}{d^{2}} \cos (\psi) R & |\psi| \leq \psi_{F o V} \\ 0 & |\psi|>\psi_{F o V}\end{cases}
$$

where $m=\frac{-\log (2)}{\log \left(\cos \left(\phi_{\frac{1}{2}}\right)\right)}$ is the order of the Lambertian emission with half irradiance at semi-angle $\phi_{\frac{1}{2}}$ (measured from the optical axis of the LED). As shown in Fig 1, $\theta$ represents the angle of irradiance, $d$ is the line-of-sight (LoS) distance between the LED and the PD, $\psi$ is the angle of incidence, $\psi_{F_{2}}$ iV is the receiver field of view (FoV) and $A_{R X}=\frac{n_{c}^{2}}{\sin ^{2}\left(\psi_{F O V}\right)} A_{P D}$ is the receiver collection area, such that $n_{c}$ is the refractive index of the optical concentrator and $A_{P D}$ is the PD area.

In most practical cases, the VLC channel is either constant (e.g., indoors VLC with no mobility) or varies very slowly compared to the transmission rate (mobility or outdoors VLC). The channel coherence time is typically 0.1 to $10 \mathrm{~ms}$ whereas the transmission rates are on the order of several tens of Mpbs to several Gbps. Thus, the channel remains constant over thousands up to millions of consecutive bits, and hence, it is considered quasi-static in the scale of interest [43].

\section{B. The MISO VLC Wiretap Channel}

We consider a typical indoor VLC scenario consisting of a room with size $L \times W \times H$, where $L, W$, and $H$ are the length, width and height of the room. As mentioned above, Alice is equipped with $N$ fixtures of LEDs and wants to communicate privately with a single-PD Bob, in the presence of a randomly located and single-PD eavesdropper (Eve) that coexists with Bob in the same room and attempts to eavesdrop

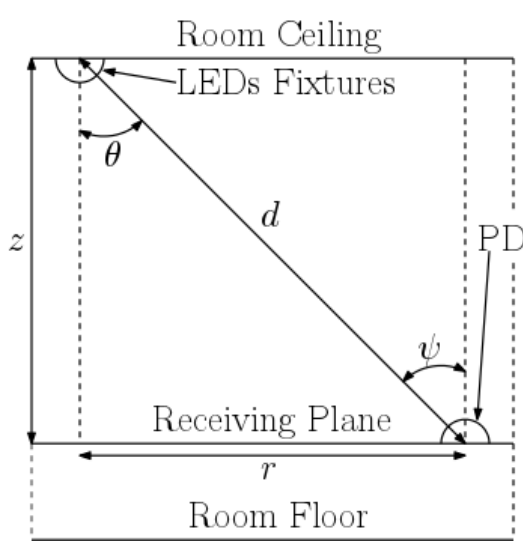

Fig. 1. VLC path gain description.

on the communication between Alice and Bob. This model is equivalent to an $N \times 1$ Gaussian MISO VLC wiretap channel. As such, the signals received at Bob and Eve are expressed, respectively, as

$$
\begin{aligned}
& y_{B}=\mathbf{h}_{B}^{T} \mathbf{s}+n_{B} \\
& y_{E}=\mathbf{h}_{E}^{T} \mathbf{s}+n_{E},
\end{aligned}
$$

where $\mathbf{h}_{B}, \mathbf{h}_{E} \in \mathbb{R}_{+}^{N}$ represent the MISO channel gain vectors of Bob and Eve, respectively, $\mathbf{s}$ is the zero-mean transmitted signal that is subject to a peak-power constraint, i.e., amplitude constraint, expressed as

$$
\|\mathbf{s}\|_{\infty} \leq A,
$$

where $A \in \mathbb{R}_{+}$is the amplitude constraint defined in subsection II-A, and $n_{B}$ and $n_{E}$ are Gaussian noise samples that are $\mathcal{N}\left(0, \sigma_{B}^{2}\right)$ and $\mathcal{N}\left(0, \sigma_{E}^{2}\right)$ distributed, respectively. Note that if the channel gain vectors $\mathbf{h}_{B}$ and $\mathbf{h}_{E}$ are colinear, the channel is equivalent to a SISO VLC wiretap channel. In this case, positive secrecy rate are guaranteed if and only if the channel is degraded. As such, the secrecy capacity is achieved by discrete input signaling [44]. To the end of the paper, we assume that $\mathbf{h}_{B}$ and $\mathbf{h}_{E}$ are not colinear.

Without loss of generality, we assume that the height of Eve is fixed and known and that its PD is facing the ceiling of the room. Let $\mathbf{h}_{E}=\left[h_{E, 1}, h_{E, 2}, \ldots, h_{E, N}\right]^{T}$. In this case, based on subsection II-A, for all $i \in \llbracket 1, N \rrbracket$, the channel coefficient $h_{E, i}$ is expressed as

$$
\begin{aligned}
h_{E, i}\left(x_{E}, y_{E}\right) & =C d_{i}\left(x_{E}, y_{E}\right)^{-(m+3)} \\
& =C\left(z^{2}+\left(x_{E}-x_{i}\right)^{2}+\left(y_{E}-y_{i}\right)^{2}\right)^{\frac{-(m+3)}{2}},
\end{aligned}
$$

where $\left(x_{E}, y_{E}\right)$ are the coordinates of Eve in the receiving plane, $\left(x_{i}, y_{i}\right)$ are the coordinates of the $i$ th fixtures of LEDs in the room's ceiling, $d_{i}$ is the LoS distance from the $i$ th fixture of LEDs to Eve, $C=\frac{\eta R T(m+1) z^{m+1} A_{R X}}{2 \pi}$ and $z$ is the vertical distance from the room ceiling to the receiving plane.

Since Eve is randomly located, the exact channel gain vector $\mathbf{h}_{E}$ is unknown to Alice. Therefore, we adopt an artificial noise based beamforming as a precoding scheme in order to degrade 
the reception of Eve. The transmitted signal is expressed, in this case, as

$$
\mathbf{s}=\mathbf{w}_{1} u+\mathbf{w}_{2} x,
$$

where $u$ is the information bearing signal, $x$ is the jamming signal and $\mathbf{w}_{1}$ and $\mathbf{w}_{2}$ are their $N \times 1$ beamforming vectors, respectively. In this scheme, Alice can exploit its perfect knowledge of Bob's CSI in designing the beamforming vector $\mathbf{w}_{2}$. In fact, Alice can transmit the artificial noise in the nullspace of Bob, i.e., $\mathbf{h}_{B}^{T} \mathbf{w}_{2}=0$, in order to cancel the interference in Bob's reception. In this case, the received signals at Bob and Eve are expressed, respectively, as

$$
\begin{aligned}
& y_{B}=\mathbf{h}_{B}^{T} \mathbf{w}_{1} u+n_{B}, \\
& y_{E}=\mathbf{h}_{E}^{T} \mathbf{w}_{1} u+\mathbf{h}_{E}^{T} \mathbf{w}_{2} x+n_{E} .
\end{aligned}
$$

Let $\rho_{B}, \rho_{E, u}$ and $\rho_{E, x}$ be defined, respectively, as

$$
\left\{\begin{array}{l}
\rho_{B} \triangleq \frac{\left(\mathbf{h}_{B}^{T} \mathbf{w}_{1}\right)^{2}}{\sigma_{B}^{2}}, \\
\rho_{E, u} \triangleq \frac{\left(\mathbf{h}_{E}^{T} \mathbf{w}_{1}\right)^{2}}{\sigma_{E}^{2}}, \quad \rho_{E, x} \triangleq \frac{\left(\mathbf{h}_{E}^{T} \mathbf{w}_{2}\right)^{2}}{\sigma_{E}^{2}}
\end{array}\right.
$$

where $\sigma_{u}^{2}$ and $\sigma_{x}^{2}$ are the variances of $u$ and $x$, respectively. Moreover, in order to satisfy the amplitude constraint in (4), we impose the following constraints on $u, x, \mathbf{w}_{1}$ and $\mathbf{w}_{2}$.

$$
\left\{\begin{array}{l}
\left\|\mathbf{w}_{1}\right\|_{\infty} \leq 1, \quad\left\|\mathbf{w}_{2}\right\|_{\infty} \leq 1, \\
|u| \leq B, \quad|x| \leq B_{c}
\end{array}\right.
$$

where $B, B_{c} \in \mathbb{R}_{+}$such that $0<B \leq A$ and $B_{c}=A-B$. Furthermore, we assume that the information-bearing signal $u$ and the jamming signal $x$ are independent, zero-mean discrete random scalar variables with finite support sets over $[-B, B]$ and $\left[-B^{c}, B^{c}\right]$, respectively. We denote by $p_{u}$ and $p_{x}$ the probability mass functions (pmf)s of $u$ and $x$, respectively.

In this paper, our objective is designing the beamformers $\mathbf{w}_{1}$ and $\mathbf{w}_{2}$ that aim to improve the secrecy performance of the MISO VLC wiretap channel in (7) when only information about the spatial distribution of Eve is known. We adopt the achievable secrecy rate as a secrecy performance measure.

\section{SeCrecy Performance Analysis}

For the MISO wiretap channel under amplitude-constrained input, the secrecy capacity achieving probability distribution is discrete with a finite support set [26]. However, the secrecy capacity, denoted by $C_{s}$, and its achieving probability distribution can be only determined numerically by invoking the same approach used in [26, Section V, PP1], since no closed-form expressions have been determined yet. In this section, we derive an achievable secrecy rate for the MISO VLC wiretap channel in (7) and we propose a low-complexity heuristic approach to derive suboptimal solutions for the best beamforming vectors.

\section{A. Achievable Secrecy Rate}

In this subsection, we derive an achievable secrecy rate for the MISO VLC wiretap channel in (7). Recall that the information-bearing signal $u$ and the jamming signal $x$ are both zero-mean and discrete with finite support sets over $[-B, B]$ and $\left[-B^{c}, B^{c}\right]$, respectively. Let $K_{u} \in \mathbb{N}^{*}$ and $K_{x} \in$ $\mathbb{N}^{*}$ be the number of mass points of $u$ and $x$, respectively. In this case, there exists $\left(u_{i}\right)_{1<i<K_{u}} \in[-B, B],\left(p_{i}\right)_{1<i<K_{u}} \in$ $[0,1],\left(x_{i}\right)_{1<i<K_{x}} \in\left[-B^{c}, \bar{B}^{c}\right]$ and $\left(q_{i}\right)_{1<i<K_{x}} \in[0,1]$, such that the pmf of $u$ and $x$ are expressed, respectively, as

$$
\begin{cases}p_{u}(z)=\sum_{i=1}^{K_{u}} p_{i} \delta\left(z-u_{i}\right), & \forall z \in[-B, B], \\ p_{x}(z)=\sum_{i=1}^{K_{x}} q_{i} \delta\left(z-x_{i}\right), & \forall z \in\left[-B^{c}, B^{c}\right] .\end{cases}
$$

Based on this, for a fixed Eve's location, an achievable secrecy rate for the MISO VLC wiretap channel in (7) is given in the following theorem.

Theorem 1. For a fixed Eve's location, an achievable secrecy rate for the MISO VLC wiretap channel in (7) is $R_{s}^{+}$, where

$$
\begin{aligned}
& R_{s}\left(\boldsymbol{w}_{1}, \boldsymbol{w}_{2}, \boldsymbol{h}_{E}\right)=\frac{1}{2} \log \left[\frac{1+2 \rho_{B} \sigma_{u}^{2}}{1+\rho_{B} \sigma_{u}^{2}}\right]-\log \left[\sum_{i, j=1}^{K} p_{i} p_{j} e^{d_{i, j}^{u}}\right] \\
& -\frac{1}{2} \log \left[1+\rho_{E, u} \sigma_{u}^{2}+\rho_{E, x} \sigma_{x}^{2}\right]+\frac{1}{2} \log \left[\frac{1+2 \rho_{E, x} \sigma_{u}^{2}}{1+\rho_{E, x} \sigma_{u}^{2}}\right] \\
& -\log \left[\sum_{i, j=1}^{K} p_{i} p_{j} e^{d_{i, j}^{x}}\right]
\end{aligned}
$$

such that

$$
\left\{\begin{array}{l}
d_{i, j}^{u}=\frac{-\rho_{B}}{4 \rho_{B} \sigma_{u}^{2}+2}\left(\rho_{B} \sigma_{u}^{2}\left(u_{i}-u_{j}\right)^{2}-2 u_{i} u_{j}\right) \\
d_{i, j}^{x}=\frac{-\rho_{E, x}}{4 \rho_{E, x} \sigma_{x}^{2}+2}\left(\rho_{E, x} \sigma_{x}^{2}\left(x_{i}-x_{j}\right)^{2}-2 x_{i} x_{j}\right) .
\end{array}\right.
$$

Proof. See appendix A.

The achievable secrecy rate $R_{s}$ is valid for any discrete random variables $u$ and $x$ and any beamforming vectors $\mathbf{w}_{1}$ and $\mathbf{w}_{2}$. However, due to the amplitude constraint imposed on the transmitted signal, the system variables $u, x, \mathbf{w}_{1}$ and $\mathbf{w}_{2}$ should satisfy the amplitude constraint in (9).

\section{B. Precoding Design}

Taking into account that Eve is randomly located, the average achievable secrecy rate $\mathbb{E}_{\mathbf{h}_{E}}\left[R_{s}\left(\mathbf{w}_{1}, \mathbf{w}_{2}, \mathbf{h}_{E}\right)\right]$ can be enhanced through a well structured design of the beamforming vectors $\mathbf{w}_{1}$ and $\mathbf{w}_{2}$. In this case, the maximum average achievable secrecy rate can be obtained through the following optimization problem.

$$
\begin{array}{r}
\mathcal{P}_{1}:\left(\mathbf{w}_{1}^{*}, \mathbf{w}_{2}^{*}\right)=\underset{\mathbf{w}_{1}, \mathbf{w}_{2} \in \mathbb{R}^{N}}{\operatorname{argmax}} \mathbb{E}_{\mathbf{h}_{E}}\left[R_{s}\left(\mathbf{w}_{1}, \mathbf{w}_{2}, \mathbf{h}_{E}\right)\right] \\
\text { s.t. }\left\{\begin{array}{l}
\mathbf{h}_{B}^{T} \mathbf{w}_{2}=0, \\
\left\|\mathbf{w}_{1}\right\|_{\infty} \leq 1, \\
\left\|\mathbf{w}_{2}\right\|_{\infty} \leq 1 .
\end{array}\right.
\end{array}
$$


The expectation $\mathbb{E}_{\mathbf{h}_{E}}\left[R_{s}\left(\mathbf{w}_{1}, \mathbf{w}_{2}, \mathbf{h}_{E}\right)\right]$ can be efficiently calculated numerically. Therefore, one can use brute-force (BF) search methods to solve problem $\mathcal{P}_{1}$. To this end, we denote by $R_{s}^{*}(B F)$, the optimal value of $\mathbb{E}_{\mathbf{h}_{E}}\left[R_{s}\left(\mathbf{w}_{1}, \mathbf{w}_{2}, \mathbf{h}_{E}\right)\right]$ obtained by $\mathrm{BF}$ search methods.

A major disadvantage of the $\mathrm{BF}$ search methods is their high computational complexity, which makes its use impracticable for low latency applications. Moreover, the expectation $\mathbb{E}_{\mathbf{h}_{E}}\left[R_{s}\left(\mathbf{w}_{1}, \mathbf{w}_{2}, \mathbf{h}_{E}\right)\right]$ does not lead to any tractable solution since it does not have a closed-form expression. This is mainly due to the complex expression of Eve's channel gain vector as shown in (5). Therefore, we propose a suboptimal and lowcomplexity approach in solving problem $\mathcal{P}_{1}$, that is detailed as follows. Let $\overline{\mathbf{h}}_{E}=\left[\bar{h}_{E, 1}, \bar{h}_{E, 2}, \ldots, \bar{h}_{E, N}\right]^{T}$ be the average channel gain vector of Eve, i.e., $\overline{\mathbf{h}}_{E} \triangleq \mathbb{E}\left[\mathbf{h}_{E}\right]$. In this case, for all $i \in \llbracket 1, N \rrbracket$, the channel coefficient $\bar{h}_{E, i}$ is expressed as

$$
\bar{h}_{E, i}=C \int_{0}^{L} \int_{0}^{W} p_{E}(x, y) d_{i}(x, y)^{-(m+3)} \mathrm{d} x \mathrm{~d} y,
$$

where $p_{E}$ is the spatial distribution of Eve within the room. Now we consider the achievable secrecy rate at average Eve's channel gain vector $R_{s}\left(\mathbf{w}_{1}, \mathbf{w}_{2}, \overline{\mathbf{h}}_{E}\right)$. Based on the expression of $d_{i, j}^{u}$ given in (12) and since for all $i \in \llbracket 1, K_{u} \rrbracket,-B \leq u_{i} \leq$ $B$, then for all $(i, j) \in \llbracket 1, K_{u} \rrbracket^{2}$, we have

$$
-\rho_{B} \sigma_{u}^{2} B^{2} \leq d_{i, j}^{u} \leq \frac{\rho_{B} \sigma_{u}^{2} B^{2}}{2 \rho_{B} \sigma_{u}^{2} B^{2}+1} .
$$

In addition, Based on the expression of $d_{i, j}^{x}$ given in (12) and since for all $i \in \llbracket 1, K_{x} \rrbracket,-B^{c} \leq x_{i} \leq B^{c}$, then for all $(i, j) \in \llbracket 1, K_{x} \rrbracket^{2}$, we have

$$
-\bar{\rho}_{E, x} \sigma_{x}^{2} B_{c}^{2} \leq d_{i, j}^{x} \leq \frac{\bar{\rho}_{E, x} \sigma_{x}^{2} B_{c}^{2}}{2 \bar{\rho}_{E, x} \sigma_{x}^{2} B_{c}^{2}+1},
$$

where $\bar{\rho}_{E, x}=\frac{\left(\overline{\mathbf{h}}_{E}^{T} \mathbf{w}_{2}\right)^{2}}{\sigma_{E}^{2}}$. Consequently, the achievable secrecy rate $R_{s}\left(\mathbf{w}_{1}, \mathbf{w}_{2}, \overline{\mathbf{h}}_{E}\right)$ is bounded as

$$
R_{s, l}\left(\mathbf{w}_{1}, \mathbf{w}_{2}, \overline{\mathbf{h}}_{E}\right) \leq R_{s}\left(\mathbf{w}_{1}, \mathbf{w}_{2}, \overline{\mathbf{h}}_{E}\right) \leq R_{s, u}\left(\mathbf{w}_{1}, \mathbf{w}_{2}, \overline{\mathbf{h}}_{E}\right),
$$

where $R_{s, l}\left(\mathbf{w}_{1}, \mathbf{w}_{2}, \overline{\mathbf{h}}_{E}\right)$ and $R_{s, u}\left(\mathbf{w}_{1}, \mathbf{w}_{2}, \overline{\mathbf{h}}_{E}\right)$ are expressed, respectively, as

$$
\left\{\begin{aligned}
R_{s, l} & \left(\mathbf{w}_{1}, \mathbf{w}_{2}, \overline{\mathbf{h}}_{E}\right)=\frac{1}{2} \log \left[\frac{1+2 \rho_{B} \sigma_{u}^{2}}{1+\rho_{B} \sigma_{u}^{2}}\right]-\frac{\rho_{B} \sigma_{u}^{2} B^{2}}{2 \rho_{B} \sigma_{u}^{2} B^{2}+1} \\
& -\frac{\bar{\rho}_{E, x} \sigma_{x}^{2} B_{c}^{2}}{2 \bar{\rho}_{E, x} \sigma_{x}^{2} B_{c}^{2}+1}-\frac{1}{2} \log \left[1+\rho_{E, u} \sigma_{u}^{2}+\rho_{E, x} \sigma_{x}^{2}\right] \\
& +\frac{1}{2} \log \left[\frac{1+2 \rho_{E, x} \sigma_{u}^{2}}{1+\rho_{E, x} \sigma_{u}^{2}}\right], \\
R_{s, u} & \left(\mathbf{w}_{1}, \mathbf{w}_{2}, \overline{\mathbf{h}}_{E}\right)=\frac{1}{2} \log \left[\frac{1+2 \rho_{B} \sigma_{u}^{2}}{1+\rho_{B} \sigma_{u}^{2}}\right]+\rho_{B} \sigma_{u}^{2} B^{2} \\
& +\bar{\rho}_{E, x} \sigma_{x}^{2} B_{c}^{2}-\frac{1}{2} \log \left[1+\rho_{E, u} \sigma_{u}^{2}+\rho_{E, x} \sigma_{x}^{2}\right] \\
& +\frac{1}{2} \log \left[\frac{1+2 \rho_{E, x} \sigma_{u}^{2}}{1+\rho_{E, x} \sigma_{u}^{2}}\right] .
\end{aligned}\right.
$$

Now we consider the two optimization problem $\mathcal{P}_{1, l}$ and $\mathcal{P}_{1, u}$ given, respectively, by

$$
\left\{\begin{aligned}
& \mathcal{P}_{1, l}: \quad\left(\mathbf{w}_{1, l}^{*}, \mathbf{w}_{2, l}^{*}\right)= \underset{\mathbf{w} \in \mathbb{R}^{N}}{\operatorname{argmax}} R_{s, l}\left(\mathbf{w}_{1}, \mathbf{w}_{2}, \overline{\mathbf{h}}_{E}\right) \\
& \text { s.t. }\left\{\begin{array}{l}
\mathbf{h}_{B}^{T} \mathbf{w}_{2}=0, \\
\left\|\mathbf{w}_{1}\right\|_{\infty} \leq 1, \\
\left\|\mathbf{w}_{2}\right\|_{\infty} \leq 1,
\end{array}\right. \\
& \mathcal{P}_{1, u}: \quad\left(\mathbf{w}_{1, u}^{*}, \mathbf{w}_{2, u}^{*}\right)=\underset{\mathbf{w} \in \mathbb{R}^{N}}{\operatorname{argmax} R_{s, u}\left(\mathbf{w}_{1}, \mathbf{w}_{2}, \overline{\mathbf{h}}_{E}\right)} \\
& \text { s.t. }\left\{\begin{array}{l}
\mathbf{h}_{B}^{T} \mathbf{w}_{2}=0, \\
\left\|\mathbf{w}_{1}\right\|_{\infty} \leq 1, \\
\left\|\mathbf{w}_{2}\right\|_{\infty} \leq 1 .
\end{array}\right.
\end{aligned}\right.
$$

Based on the above, our approach in solving problem $\mathcal{P}_{1}$ is as follows. First, we solve problems $\mathcal{P}_{1, l}$ and $\mathcal{P}_{1, u}$. Let $\left(\mathbf{w}_{1, l}^{*}, \mathbf{w}_{2, l}^{*}\right)$ and $\left(\mathbf{w}_{1, u}^{*}, \mathbf{w}_{2, u}^{*}\right)$ be their solutions, respectively. Then we inject the obtained solutions into the expression of $\mathbb{E}_{\mathbf{h}_{E}}\left[R_{s}\left(\mathbf{w}_{1}, \mathbf{w}_{2}, \mathbf{h}_{E}\right)\right]$ and we select the maximum value between them. Precisely, let $R_{s, l}^{*} \triangleq \mathbb{E}_{\mathbf{h}_{E}}\left[R_{s}\left(\mathbf{w}_{1, l}^{*}, \mathbf{w}_{2, l}^{*}, \mathbf{h}_{E}\right)\right]$ and $R_{s, u}^{*} \triangleq \mathbb{E}_{\mathbf{h}_{E}}\left[R_{s}\left(\mathbf{w}_{1, u}^{*}, \mathbf{w}_{2, u}^{*}, \mathbf{h}_{E}\right)\right]$. Thus, the suboptimal average achievable secrecy rate, obtained by our proposed scheme (PS), is expressed as ${ }^{2}$

$$
R_{s}^{*}(P S)=\max \left(R_{s, l}^{*}, R_{s, u}^{*}\right) .
$$

In the following, we start by solving problem $\mathcal{P}_{1, l}$ and then we consider problem $\mathcal{P}_{1, u}$.

\section{1) Optimization Problem $\mathcal{P}_{1, l}$ :}

Let $\mathbf{W} \triangleq\left[\mathbf{w}_{1}, \mathbf{w}_{2}\right]$ be the precoding matrix of the system and consider the change of optimization variable given by $\mathbf{W}=\mathbf{B}^{\perp} \sqrt{\mathbf{X}}$, where $\mathbf{B}$ is the $2 \times N$ matrix expressed as $\mathbf{B}=\left[\frac{\mathbf{h}_{B}}{\sigma_{B}} \frac{\mathbf{h}_{E}}{\sigma_{E}}\right]^{T}$. Thus, $\mathbf{X}$ is a $2 \times 2$ matrix expressed as

$$
\mathbf{X}=\left[\begin{array}{ll}
x_{1} & x_{2} \\
x_{3} & x_{4}
\end{array}\right]=\left[\begin{array}{ll}
\frac{\left(\mathbf{h}_{B}^{T} \mathbf{w}_{1}\right)^{2}}{\sigma_{B}^{2}} & \frac{\left(\mathbf{h}_{B}^{T} \mathbf{w}_{2}\right)^{2}}{\sigma_{B}^{2}} \\
\frac{\left(\mathbf{h}_{E}^{T} \mathbf{w}_{1}\right)^{2}}{\sigma_{E}^{2}} & \frac{\left(\mathbf{h}_{E}^{T} \mathbf{w}_{2}\right)^{2}}{\sigma_{E}^{2}}
\end{array}\right] .
$$

On the other hand, note that the infinity norm constraints $\left\|\mathbf{w}_{1}\right\|_{\infty} \leq 1$ and $\left\|\mathbf{w}_{2}\right\|_{\infty} \leq 1$ imposed to the beamforming vectors are equivalent to imposing a unit infinity norm constraint on the hermitian of the precoding matrix $\mathbf{W}$, i.e.,

$$
\left\|\mathbf{W}^{T}\right\|_{\infty} \leq 1
$$

In order to satisfy this constraint, we impose the following infinity norm constraint on the matrix $\mathbf{X}$.

$$
\left\|\sqrt{\mathbf{X}}^{T}\right\|_{\infty} \leq A_{\mathbf{B}}
$$

where $A_{\mathbf{B}}=\min \left(\left\|\mathbf{B}^{T}\right\|_{\infty}, \frac{1}{\left\|\left(\mathbf{B}^{\perp}\right)^{T}\right\|_{\infty}}\right)$. In this case, the infinity norm constraint in (22) and the infinity norm constraint in (23) are equivalent, i.e., if one is satisfied, the other is

${ }^{2}$ Since the secrecy rate $\mathbb{E}_{\mathbf{h}_{E}}\left[R_{s}\left(\mathbf{w}_{1}, \mathbf{w}_{2}, \mathbf{h}_{E}\right)\right]$ is achievable for any beamforming vectors $\left(\mathbf{w}_{1}, \mathbf{w}_{2}\right)$, both $R_{s, l}^{*}$ and $R_{s, u}^{*}$ are achievable, which implies that the secrecy rate $R_{s}^{*}(P S)=\max \left(R_{s, l}^{*}, R_{s, u}^{*}\right)$ is also achievable. 
automatically satisfied. In fact, if the constraint in (22) is satisfied, then

$$
\begin{aligned}
\left\|\sqrt{\mathbf{x}}^{T}\right\|_{\infty}=\left\|\mathbf{W}^{T} \mathbf{B}^{T}\right\|_{\infty} & \leq\left\|\mathbf{w}^{T}\right\|_{\infty}\left\|\mathbf{B}^{T}\right\|_{\infty} \\
& \leq\left\|\mathbf{B}^{T}\right\|_{\infty} \\
& \leq A_{\mathbf{B}},
\end{aligned}
$$

and if the constraint in (23) is satisfied, then

$$
\begin{aligned}
\left\|\mathbf{W}^{T}\right\|_{\infty}=\left\|\sqrt{\mathbf{x}}^{T}\left(\mathbf{B}^{\perp}\right)^{T}\right\|_{\infty} & \leq\left\|\sqrt{\mathbf{X}}^{T}\right\|_{\infty}\left\|\left(\mathbf{B}^{\perp}\right)^{T}\right\|_{\infty} \\
& \leq \min \left(\left\|\mathbf{B}^{\perp}\right\|_{\infty}\|\mathbf{B}\|_{\infty}, 1\right) \\
& \leq 1 .
\end{aligned}
$$

Now let $\mathbf{x}$ be the vector form of the matrix $\mathbf{X}$, given by $\mathbf{x}=$ $\left[x_{1}, x_{2}, x_{3}, x_{4}\right]^{T}$ and consider the functions $f$, expressed for all $\mathbf{x} \in \mathbb{R}_{+}^{4}$, as

$$
\begin{aligned}
f(\mathbf{x}) & =-\frac{1}{2} \log \left[\frac{1+2 x_{1} \sigma_{u}^{2}}{1+x_{1} \sigma_{u}^{2}}\right]+\frac{x_{1} \sigma_{u}^{2} B^{2}}{2 x_{1} \sigma_{u}^{2} B^{2}+1} \\
& +\frac{1}{2} \log \left[1+x_{3} \sigma_{u}^{2}+x_{4} \sigma_{x}^{2}\right]-\frac{1}{2} \log \left[\frac{1+2 x_{4} \sigma_{u}^{2}}{1+x_{4} \sigma_{u}^{2}}\right] \\
& +\frac{x_{4} \sigma_{x}^{2} B_{c}^{2}}{2 x_{4} \sigma_{x}^{2} B_{c}^{2}+1} .
\end{aligned}
$$

Moreover, consider the functions $g, h$ and $k$, expressed, for all $\mathbf{x} \in \mathbb{R}_{+}^{4}$, as

$$
\left\{\begin{array}{l}
g(\mathbf{x})=x_{2} \\
h(\mathbf{x})=\sqrt{x_{1}}+\sqrt{x_{3}}-A_{\mathbf{B}}, \\
k(\mathbf{x})=\sqrt{x_{4}}-A_{\mathbf{B}}
\end{array}\right.
$$

Based on the above, problem $\mathcal{P}_{1, l}$ can be rewritten as

$$
\begin{array}{r}
\mathcal{P}_{1, l}: \quad \mathbf{x}^{*}=\underset{\mathbf{x} \in \mathbb{R}_{+}^{4}}{\operatorname{argmin}} f(\mathbf{x}) \\
\text { s.t. }\left\{\begin{array}{l}
g(\mathbf{x})=0, \\
h(\mathbf{x}) \leq 0, \\
k(\mathbf{x}) \leq 0 .
\end{array}\right.
\end{array}
$$

The function $g$ is convex. On the other hand, for all $\mathbf{x} \in \mathbb{R}_{+}^{4}$, we have $f(\mathbf{x})=f_{1}(\mathbf{x})-f_{2}(\mathbf{x})$, where

$$
\left\{\begin{aligned}
f_{1}(\mathbf{x}) & =-\frac{1}{2} \log \left[\frac{1+2 \sigma_{s}^{2} x_{1}}{1+\sigma_{s}^{2} x_{1}}\right]-\frac{1}{2} \log \left[\frac{1+2 x_{4} \sigma_{u}^{2}}{1+x_{4} \sigma_{u}^{2}}\right], \\
f_{2}(\mathbf{x}) & =-\frac{x_{1} \sigma_{u}^{2} B^{2}}{2 x_{1} \sigma_{u}^{2} B^{2}+1}-\frac{1}{2} \log \left[1+x_{3} \sigma_{u}^{2}+x_{4} \sigma_{x}^{2}\right] \\
& -\frac{x_{4} \sigma_{x}^{2} B_{c}^{2}}{2 x_{4} \sigma_{x}^{2} B_{c}^{2}+1} .
\end{aligned}\right.
$$

In addition, for all $\mathbf{x} \in \mathbb{R}_{+}^{4}$, we have $h(\mathbf{x})=h_{1}(\mathbf{x})-h_{2}(\mathbf{x})$ and $k(\mathbf{x})=k_{1}(\mathbf{x})-k_{2}(\mathbf{x})$, where for all $\mathbf{x} \in \mathbb{R}_{+}^{4}$

$$
\begin{cases}h_{1}(\mathbf{x})=-A_{\mathbf{B}}, & h_{2}(\mathbf{x})=-\sqrt{x_{1}}-\sqrt{x_{3}}, \\ k_{2}(\mathbf{x})=-A_{\mathbf{B}}, & k_{2}(\mathbf{x})=-\sqrt{x_{4}} .\end{cases}
$$

Clearly, the functions $f_{1}, f_{2}, h_{1}, h_{2}, k_{1}$ and $k_{2}$ are all convex and, therefore, the functions $f, h$ and $k$ are each a difference of two convex functions. In this case, problem $\mathcal{P}_{1, l}$ is a difference of convex (DC) problem. Therefore, it is typical to use the convex-concave procedure (CCP) proposed in [45] in solving problem $\mathcal{P}_{1, l}$. As such, we convexify the functions $f$, $h$ and $k$ through a simple linearization of the functions $f_{2}, h_{2}$ and $k_{2}$, respectively, by applying the first-order Taylor series approximation around a given point $x_{l} \in \mathbb{R}_{+}^{4}$. Consequently, the convex form of $f, h$ and $k$, denoted respectively by $\tilde{f}, \tilde{h}$ and $\tilde{k}$, are expressed, respectively, as

$$
\left\{\begin{array}{l}
\tilde{f}\left(\mathbf{x}, \mathbf{x}_{j}\right)=f_{1}(\mathbf{x})-f_{2}\left(\mathbf{x}_{j}\right)-\nabla_{f_{2}}\left(\mathbf{x}_{j}\right)^{T}\left(\mathbf{x}-\mathbf{x}_{j}\right), \\
\tilde{h}\left(\mathbf{x}, \mathbf{x}_{j}\right)=h_{1}(\mathbf{x})-h_{2}\left(\mathbf{x}_{j}\right)-\nabla_{h_{2}}\left(\mathbf{x}_{j}\right)^{T}\left(\mathbf{x}-\mathbf{x}_{j}\right), \\
\tilde{k}\left(\mathbf{x}, \mathbf{x}_{j}\right)=k_{1}(\mathbf{x})-k_{2}\left(\mathbf{x}_{j}\right)-\nabla_{k_{2}}\left(\mathbf{x}_{j}\right)^{T}\left(\mathbf{x}-\mathbf{x}_{j}\right),
\end{array}\right.
$$

where $\nabla_{f_{2}}(\mathbf{x}), \nabla_{h_{2}}(\mathbf{x})$ and $\nabla_{k_{2}}(\mathbf{x})$ are the gradients of the functions $f_{2}, h_{2}$ and $k_{2}$, respectively. Furthermore, for all $\mathbf{x} \in$ $\mathbb{R}_{+}^{4}, \nabla_{f_{2}}(\mathbf{x})$ is expressed as $\nabla_{f_{2}}(\mathbf{x})=\left[v_{1}, v_{2}, v_{3}, v_{4}\right]^{T}$, where

$$
\left\{\begin{array}{l}
v_{1}=-\frac{\sigma_{u}^{2} B^{2}}{\left(2 \sigma_{u}^{2} B^{2} x_{1}+1\right)^{2}}, \\
v_{2}=0, \\
v_{3}=-\frac{\sigma_{u}^{2}}{2\left(1+\sigma_{u}^{2} x_{3}+\sigma_{x}^{2} x_{4}\right)}, \\
v_{4}=-\frac{\sigma_{x}^{2}}{2\left(1+\sigma_{u}^{2} x_{3}+\sigma_{x}^{2} x_{4}\right)}-\frac{\sigma_{x}^{2} B_{c}^{2}}{\left(2 \sigma_{x}^{2} B_{c}^{2} x_{4}+1\right)^{2}} .
\end{array}\right.
$$

In addition, $\nabla_{h_{2}}(\mathbf{x})$ and $\nabla_{k_{2}}(\mathbf{x})$ are expressed respectively, for all $\mathbf{x} \in \mathbb{R}_{+}^{4}$, as

$$
\left\{\begin{array}{l}
\nabla_{h_{2}}(\mathbf{x})=\left[-\frac{1}{2 \sqrt{x_{1}}}, 0,-\frac{1}{2 \sqrt{x_{3}}}, 0\right]^{T}, \\
\nabla_{k_{2}}(\mathbf{x})=\left[0,0,0-\frac{1}{2 \sqrt{x_{4}}}\right]^{T} .
\end{array}\right.
$$

Consequently, armed with the above, the convex form of problem $\mathcal{P}_{1, l}$ is given by

$$
\begin{array}{r}
\mathcal{P}_{1, l}^{\prime}\left(\mathbf{x}_{j}\right): \quad \mathbf{x}^{*}=\underset{\mathbf{x} \in \mathbb{R}_{+}^{4}}{\operatorname{argmin}} \tilde{f}\left(\mathbf{x}, \mathbf{x}_{j}\right) \\
\text { s.t. }\left\{\begin{array}{l}
g(\mathbf{x})=0, \\
\tilde{h}\left(\mathbf{x}, \mathbf{x}_{j}\right) \leq 0, \\
\tilde{k}\left(\mathbf{x}, \mathbf{x}_{j}\right) \leq 0 .
\end{array}\right.
\end{array}
$$

Problem $\mathcal{P}_{1, l}^{\prime}\left(\mathbf{x}_{j}\right)$ is a convex optimization problem that depends on the linearization point $\mathbf{x}_{j}$ and that can be solved efficiently using standard optimization packages [46], [47].

Based on the above analysis, the detailed iterative algorithm for solving problem $\mathcal{P}_{1, l}$ is given in Algorithm 1 on top of next page, where the initial point $\mathbf{x}_{0}$ is a random feasible point that satisfies the constraints of problem $\mathcal{P}_{1, l}$ in (26), $\epsilon$ is a fixed relative error between two consecutive iterations and $L$ is the maximum number of iteration. Note that it was shown in [45] and references therein that CCP is an efficient method in solving DC problems, where a complete proof of convergence was provided. Finally, after obtaining the solution $\mathbf{x}^{*}$ from Algorithm 1, we formulate the best matrix $\mathbf{X}^{*}$ and we determine the best beamforming vectors as $\left[\mathbf{w}_{1, l}^{*}, \mathbf{w}_{2, l}^{*}\right]=\mathbf{B}^{\perp} \mathbf{X}^{*}$. 


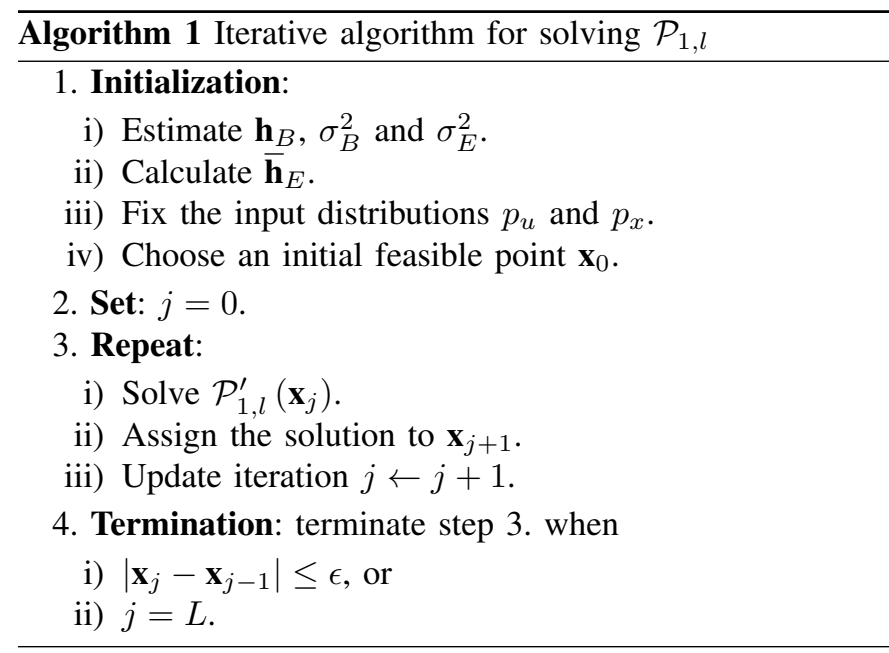

2) Optimization Problem $\mathcal{P}_{1, u}$ :

Using the same notations and change of variable adopted in the previous paragraph, problem $\mathcal{P}_{1, u}$ can be rewritten as

$$
\begin{array}{r}
\mathcal{P}_{1, l}: \quad \mathbf{x}^{*}=\underset{\mathbf{x} \in \mathbb{R}_{+}^{4}}{\operatorname{argmin}} q(\mathbf{x}) \\
\text { s.t. }\left\{\begin{array}{l}
g(\mathbf{x})=0, \\
h(\mathbf{x}) \leq 0, \\
k(\mathbf{x}) \leq 0 .
\end{array}\right.
\end{array}
$$

where $q$ is the function expressed, for all $\mathbf{x} \in \mathbb{R}^{4}$, as

$$
\begin{aligned}
q(\mathbf{x}) & =-\frac{1}{2} \log \left[\frac{1+2 x_{1} \sigma_{u}^{2}}{1+x_{1} \sigma_{u}^{2}}\right]-x_{1} \sigma_{u}^{2} B^{2} \\
& +\frac{1}{2} \log \left[1+x_{3} \sigma_{u}^{2}+x_{4} \sigma_{x}^{2}\right]-\frac{1}{2} \log \left[\frac{1+2 x_{4} \sigma_{u}^{2}}{1+x_{4} \sigma_{u}^{2}}\right] \\
& -x_{4} \sigma_{u}^{2} B^{2} .
\end{aligned}
$$

Note that, for all $\mathbf{x} \in \mathbb{R}_{+}^{4}$, we have $q(\mathbf{x})=q_{1}(\mathbf{x})-q_{2}(\mathbf{x})$, where

$$
\left\{\begin{aligned}
q_{1}(\mathbf{x}) & =-\frac{1}{2} \log \left[\frac{1+2 \sigma_{u}^{2} x_{1}}{1+\sigma_{u}^{2} x_{1}}\right]-x_{1} \sigma_{u}^{2} B^{2}-x_{4} \sigma_{x}^{2} B_{c}^{2} \\
& -\frac{1}{2} \log \left[\frac{1+2 x_{4} \sigma_{x}^{2}}{1+x_{4} \sigma_{x}^{2}}\right] \\
q_{2}(\mathbf{x}) & =-\frac{1}{2} \log \left[1+x_{3} \sigma_{u}^{2}+x_{4} \sigma_{x}^{2}\right] .
\end{aligned}\right.
$$

Clearly, the functions $q_{1}$ and $q_{2}$ are convex and, therefore, the function $q$ is a difference of two convex functions. Therefore, problem $\mathcal{P}_{1, u}$ is a difference of convex (DC) problem. To tackle this problem, we use the CCP and we convexify the function $q$ through a simple linearization of the function $q_{2}$ by applying the first-order Taylor series approximation around a given point $x_{j}$. Consequently, the convex form of $q$, denoted by $\tilde{g}$ is expressed, as

$$
\tilde{q}\left(\mathbf{x}, \mathbf{x}_{j}\right)=q_{1}(\mathbf{x})-q_{2}\left(\mathbf{x}_{j}\right)-\nabla_{q_{2}}\left(\mathbf{x}_{j}\right)^{T}\left(\mathbf{x}-\mathbf{x}_{j}\right),
$$

where $\nabla_{q_{2}}(\mathbf{x})$ is the gradient of the function $q_{2}$ which is expressed, for all $\mathbf{x} \in \mathbb{R}_{+}^{4}$, as

$$
\nabla_{q_{2}}(\mathbf{x})=\left[0,0, \frac{\sigma_{u}^{2} / 2}{1+\sigma_{u}^{2} x_{3}+\sigma_{x}^{2} x_{4}}, \frac{\sigma_{x}^{2} / 2}{1+\sigma_{u}^{2} x_{3}+\sigma_{x}^{2} x_{4}}\right]^{T} .
$$

Consequently, armed with the above, the convex form of problem $\mathcal{P}_{1, u}$ is given by

$$
\begin{array}{r}
\mathcal{P}_{1, u}^{\prime}\left(\mathbf{x}_{j}\right): \quad \mathbf{x}^{*}=\underset{\mathbf{x} \in \mathbb{R}_{+}^{4}}{\operatorname{argmin}} \tilde{q}\left(\mathbf{x}, \mathbf{x}_{j}\right) \\
\text { s.t. }\left\{\begin{array}{l}
g(\mathbf{x})=0, \\
\tilde{h}\left(\mathbf{x}, \mathbf{x}_{j}\right) \leq 0, \\
\tilde{k}\left(\mathbf{x}, \mathbf{x}_{j}\right) \leq 0 .
\end{array}\right.
\end{array}
$$

Problem $\mathcal{P}_{1, u}^{\prime}\left(\mathbf{x}_{j}\right)$ is a convex optimization problem that can be solved efficiently using standard optimization packages [46], [47]. Therefore, the detailed iterative algorithm for solving $\mathcal{P}_{1, u}$ can be given as in Algorithm 1, where it suffices to substitute $\mathcal{P}_{1, l}^{\prime}$ by $\mathcal{P}_{1, u}^{\prime}$ and choose $\mathbf{x}_{0}$ as any random feasible point that satisfies the constraints of problem $\mathcal{P}_{1, u}$. Finally, after obtaining the solution $\mathbf{x}^{*}$ from Algorithm 1, we formulate the best matrix $\mathbf{X}^{*}$ and we determine the best beamforming vectors as $\left[\mathbf{w}_{1, u}^{*}, \mathbf{w}_{2, u}^{*}\right]=\mathbf{B}^{\perp} \mathbf{X}^{*}$.

\section{Complexity Analysis}

In this part, we evaluate the computational complexity of the proposed precoding scheme. In Algorithm 1, we employ the well known interior point algorithm (IPA) in solving the invoked convex problem. Therefore, we employ the number of Newton steps, denoted by $N_{s}$, as a complexity measure. The number of Newton steps denotes the number of recursive iterations till convergence from a given starting point, i.e., the number of required recursive steps to reach a local solution. Based on [48], the worst-case $N_{s}$ to reach a local solution in a non-linear convex problem is expressed as

$$
N_{s} \sim \sqrt{\text { problem size }}
$$

where the problem size is the number of optimization scalar variables. The size of the optimization variable $\mathbf{x}$ adopted in the previous subsection is equal to 4 . Moreover, Algorithm 1 solves a non-linear convex problem at most $L$-times, and thus, it employs the IPA at most $L$-times. Based on this, the worst-case complexity of our proposed scheme is given by

$$
\begin{aligned}
N_{s} & =N_{s}\left(\mathcal{P}_{1, l}\right)+\left(\mathcal{P}_{1, u}\right) \\
& \sim 4 L+4 L \\
& \sim 8 L .
\end{aligned}
$$

Therefore, it can be seen that the worst-case complexity of our precoding scheme is a linear function of the maximum number of iteration $L$.

\section{Input Distributions}

The achievable secrecy rates derived in subsection III-B is valid for any bounded discrete distributions $p_{u}$ and $p_{x}$. In this work, we adopt the truncated discrete generalized normal distribution (TDGN) as probability distribution for $u$ and $x$. 
The motivation behind using this family of distributions is that it extends and generalizes various discrete probability distributions. In fact, let $D \in \mathbb{R}_{+}^{*}$ and let $v$ be a discrete random scalar variable satisfying $|v| \leq D$. In addition, let $K \in \mathbb{N}^{*}$ be the number of mass points of $v$ and let $\left(v_{i}\right)_{1 \leq i \leq K} \in[-D, D]$ and $\left(r_{i}\right)_{1 \leq i \leq K} \in[0,1]$ be its sets of mass points and mass probabilities, respectively. Based on this, the pmf of $v$ is expressed, for all $z \in \mathbb{R}$, as

$$
p_{v}(z)=\sum_{i=1}^{K} r_{i} \delta\left(z-v_{i}\right) .
$$

In this case, $v$ follows a TDGN distribution over $[-D, D]$ denoted by $\operatorname{TDGN}(K, D, \alpha, \beta)$, where $\alpha \in \mathbb{R}_{+}^{*}$ represents its scale parameter and $\beta \in \mathbb{R}_{+}^{*}$ represents its shape parameter, if for all $i \in \llbracket 1, K \rrbracket, v_{i}=\frac{2 i-K_{-} 1}{K_{-} 1} D$ and $r_{i}=\frac{r_{i}^{\prime}}{r_{T}}$, such that

$$
r_{i}^{\prime}=\frac{\beta}{2 \alpha \Gamma\left(\frac{1}{\beta}\right)} e^{-\left(\frac{\left|v_{i}\right|}{\alpha}\right)^{\beta}},
$$

and $r_{T}=\sum_{i=1}^{K} r_{i}^{\prime}$, where $\Gamma(\cdot)$ denotes the Gamma function. Note that, based on (44) and according to the parameters $\alpha$ and $\beta$, the TDGN distribution includes:

- The Dirac distribution when $\alpha \rightarrow 0$ and $\beta \rightarrow 0$.

- The truncated discrete Laplace distribution when $\beta=1$.

- The truncated discrete Gaussian distribution when $\beta=2$.

- The truncated discrete uniform distribution when $\alpha \rightarrow \infty$ and $\beta \rightarrow \infty$.

Consequently, by adopting the TDGN distribution as an input signaling scheme, the secrecy performance of the system can be enhanced through an optimal design of the shape and the scale parameters as well as the number of mass points. In this context, we assume that $u$ and $x$ follow $\operatorname{TDGN}\left(K_{u}, B, \alpha_{u}, \beta_{u}\right)$ and $\operatorname{TDGN}\left(K_{x}, B_{c}, \alpha_{x}, \beta_{x}\right)$, respectively, where $K_{u}, K_{x} \in$ $\mathbb{N}^{*}$ and $\left(\alpha_{u}, \beta_{u}, \alpha_{x}, \beta_{x}\right) \in \mathbb{R}_{+}^{*}$.

\section{E. Average Upper Bound}

When the location of Eve is fixed, an upper bound on the secrecy capacity of the MISO VLC wiretap channel in (3) may be obtained by converting the amplitude constraint in (4) into an average power constraint as

$$
\|\mathbf{s}\|_{\infty} \leq A \Longrightarrow \operatorname{Tr}\left(\mathbf{K}_{\mathbf{s}}\right)=\mathbb{E}\left(\|\mathbf{s}\|_{2}^{2}\right) \leq N A^{2}
$$

which is the trace constraint on the input covariance. Consequently, by relaxing the amplitude constraint and only considering the trace constraint, we only enhance the secrecy capacity and, thus, the average secrecy capacity of the new MISO VLC wiretap channel is an upper bound on that of the original wiretap channel. However, the secrecy capacity of the new wiretap channel is known and Gaussian is optimal. Consequently, the upper bound is given by

$$
\begin{aligned}
R_{u}\left(\mathbf{h}_{E}\right)=\max _{\mathbf{K}_{s} \geq 0} \frac{1}{2} \log \left[\frac{1+\frac{\mathbf{h}_{B}^{T} \mathbf{K}_{\mathbf{s}} \mathbf{h}_{B}}{\sigma_{B}^{2}}}{1+\frac{\mathbf{h}_{E}^{T} \mathbf{K}_{\mathbf{s}} \mathbf{h}_{E}}{\sigma_{E}^{2}}}\right] \\
\text { s.t. } \operatorname{Tr}\left(\mathbf{K}_{\mathbf{s}}\right) \leq N A^{2},
\end{aligned}
$$

Based on the results of [49], the optimal covariance matrix of (46) is given through the active eigenvectors of the matrix $\frac{1}{\sigma_{B}^{2}} \mathbf{h}_{B} \mathbf{h}_{B}^{T}-\frac{1}{\sigma_{E}^{2}} \mathbf{h}_{E} \mathbf{h}_{E}^{T}$, i.e., the eigenvectors associated to the positive eigenvalues of this matrix. In the following lemma, we characterize the active eigenvectors of the matrix $\frac{1}{\sigma_{B}^{2}} \mathbf{h}_{B} \mathbf{h}_{B}^{T}-$ $\frac{1}{\sigma_{E}^{2}} \mathbf{h}_{E} \mathbf{h}_{E}^{T}$.

Lemma 1. The matrix $\frac{1}{\sigma_{B}^{2}} \boldsymbol{h}_{B} \boldsymbol{h}_{B}^{T}-\frac{1}{\sigma_{E}^{2}} \boldsymbol{h}_{E} \boldsymbol{h}_{E}^{T}$ has only one active orthonormal eigenvector, denoted by $\boldsymbol{e}\left(\boldsymbol{h}_{E}\right)$.

Proof. See appendix B.

A full characterization of the spectrum of the matrix $\frac{1}{\sigma_{B}^{2}} \mathbf{h}_{B} \mathbf{h}_{B}^{T}-\frac{1}{\sigma_{E}^{2}} \mathbf{h}_{E} \mathbf{h}_{E}^{T}$ is provided in the proof of lemma 1, where closed-form expressions of its eigenvectors and their associated eigenvalues are derived. Now based on lemma 1, the upper bound $R_{u}\left(\mathbf{h}_{E}\right)$ is expressed as

$$
R_{u}\left(\mathbf{h}_{E}\right)=\frac{1}{2} \log \left[\frac{1+\frac{N A^{2}}{\sigma_{B}^{2}}\left(\mathbf{h}_{B}^{T} \mathbf{e}\left(\mathbf{h}_{E}\right)\right)^{2}}{1+\frac{N A^{2}}{\sigma_{E}^{2}}\left(\mathbf{h}_{E}^{T} \mathbf{e}\left(\mathbf{h}_{E}\right)\right)^{2}}\right],
$$

and since Eve is randomly located within the room, an average upper bound on the secrecy capacity of the system is given by

$$
R_{u}^{*}=\mathbb{E}_{\mathbf{h}_{E}}\left[R_{u}\left(\mathbf{h}_{E}\right)\right]
$$

The expectation in (48) can be efficiently determined numerically using any mathematical software.

\section{Simulation Results}

\section{A. Simulation Overview}

In this section, our purpose is to simulate the average secrecy rates derived in the previous section and compare them with their respective theoretical expressions. The theoretical and simulated average secrecy rates are detailed as follows.

1) For the average achievable secrecy rate, we simulated the average secrecy rate for both cases, namely, the one obtained from the BF search method and the one obtained from our proposed scheme (PS). These two cases are detailed as follows.

- For the BF search method, the theoretical result is $R_{s}^{*}(B F)$, which is obtained by solving problem $\mathcal{P}_{1}$ in (13), whereas the simulation result is obtained by injecting the obtained solution from (13) into $R_{s}\left(\mathbf{w}_{1}, \mathbf{w}_{2}, \mathbf{h}_{E}\right)$ for every Eve's channel realization $\mathbf{h}_{E}$ and then taking the average.

- For the PS, the theoretical result is obtained from the expression of $R_{s}^{*}(P S)$ in (20), whereas the simulation results are obtained from injecting the solutions of $R_{s}^{*}(P S)$ into $R_{s}\left(\mathbf{w}_{1}, \mathbf{w}_{2}, \mathbf{h}_{E}\right)$ for every Eve's channel realization $\mathbf{h}_{E}$ and then taking the average.

2) For the secrecy capacity, and since there is no closedform expression, we computed the secrecy capacity of the system numerically, by invoking the same approach used in [26, Section V, PP1], for every Eve's channel realization $\mathbf{h}_{E}$ and then taking the average.

3) For the upper bound, the theoretical expression is given in (48). For the simulation, we compute the expression 


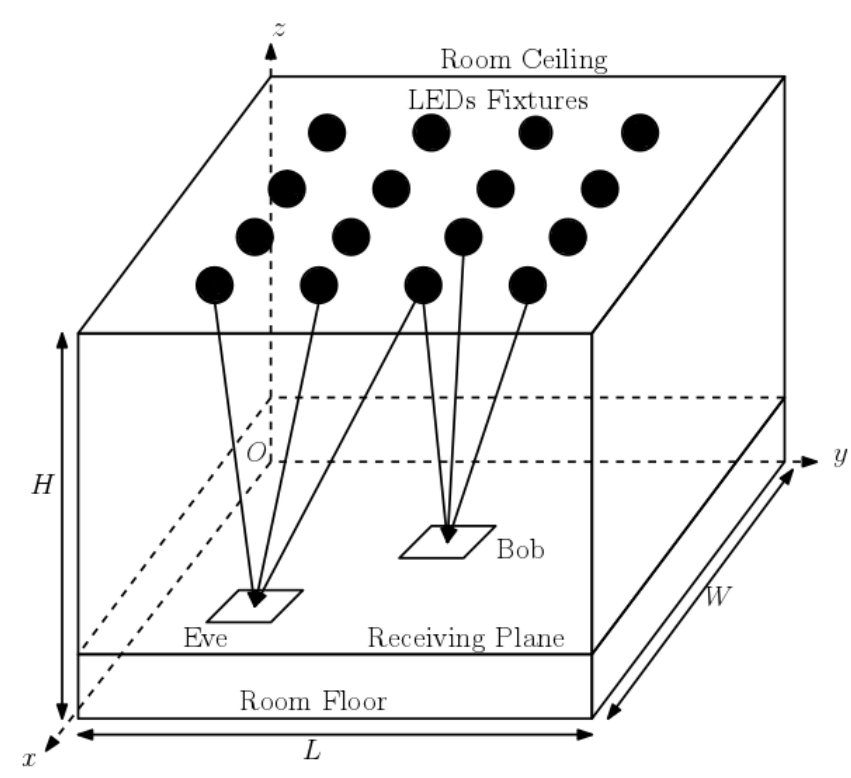

Fig. 2. A MISO VLC system with $N=16$ fixtures of LEDs.

of the upper bound in (47) for every Eve's channel realization $\mathbf{h}_{E}$ and then taking the average.

\section{B. Simulation Settings}

We considered a typical indoor VLC scenario, which is widely adopted in the literature [27]. We considered a single room with size $L \times W \times H=5 \mathrm{~m} \times 5 \mathrm{~m} \times 4 \mathrm{~m}$ as shown in Fig. 2. A Cartesian coordinate system, shown in Fig. 2, is used. The parameters of the room, the transmitter and the two receivers are given in Table I. The fixtures of LEDs of Alice are located in the ceiling of the room, where their number and their positions in the horizontal plane are shown in Table II. The receivers height measured from the room's floor is $1 \mathrm{~m}$. For simplicity, we let $B=B_{c}=\frac{A}{2}$ and the information bearing signal $u$ and the jamming signal $x$ be identically distributed. The average noise powers at Bob at Eve are $\sigma_{B}^{2}=\sigma_{E}^{2}=\sigma^{2}$, where $\sigma^{2}$ will be defined in the following section. The simulation results are obtained through $10^{5}$ independents Monte Carlo trials on the location of Eve within the room. For this case, the spatial distribution of Eve within the room is uniform, i.e., $p_{E}(x, y)=\frac{1}{L \times H}$. We use $\epsilon=10^{-3}$ and $L=10$ as stopping criterion for Algorithm 1.

\section{Numerical Results}

Fig. 3 presents the average upper bound $R_{u}^{*}$, the average secrecy capacity $C_{S}$, the average achievable secrecy rate $R_{s}^{*}(B F)$ and $R_{s}^{*}(P S)$, versus the square of the amplitude constraint $A^{2}$ in $\mathrm{dBm}$, for the average noise powers $\sigma^{2}=$ $\sigma_{1}^{2}=-98.82 \mathrm{dBm}$ and $\sigma^{2}=\sigma_{2}^{2}=-68.82 \mathrm{dBm}$ and for the numbers of Fixtures of LEDs at Alice $N=16$ and $N=4$. For the proposed scheme, the case of transmission without AN is also presented, which is obtained by setting $\rho_{E, x}=0$ and $|u| \leq A$. The legitimate receiver Bob is located at the center of the room. Fig. 3 shows that there is a small gap in the secrecy
TABLE I

MISO VLC SYSTEM PARAMETERS

\begin{tabular}{|l|c|}
\hline \multicolumn{2}{|c|}{ Transmitter configuration } \\
\hline Number of LEDs per fixture & 6 \\
\hline Angle of irradiance $\theta$ & $60^{\circ}$ \\
\hline LED conversion factor $\eta$ & $0.44 \mathrm{~W} / \mathrm{A}$ \\
\hline DC-offset current $I_{D C}$ & $700 \mathrm{~mA}$ \\
\hline Modulation index $\nu$ & 0.2 \\
\hline \multicolumn{2}{|c|}{ Receivers configuration } \\
\hline \hline PD geometric area $A_{P D}$ & $1 \mathrm{~cm}^{2}$ \\
\hline PD responsivity $R$ & $0.54 \mathrm{~A} / \mathrm{W}$ \\
\hline PD field of view $\Psi_{F O V}$ & $60^{\circ}$ \\
\hline Optical concentrator refractive index $n$ & 1.5 \\
\hline Transimpedance filter gain $T$ & 1 \\
\hline
\end{tabular}

TABLE II

POSITIONS OF THE LEDS FIXTURES

\begin{tabular}{|c|l|c|c|c|c|c|c|c|c|}
\hline \multirow{2}{*}{$\mathrm{N}=4$} & Fixtures & \multicolumn{2}{|c|}{1} & \multicolumn{2}{c|}{2} & \multicolumn{2}{|c|}{3} & \multicolumn{2}{c|}{4} \\
\cline { 2 - 10 } & Coordinates & \multicolumn{2}{|c|}{$(1,1)$} & \multicolumn{2}{|c|}{$(1,4)$} & \multicolumn{2}{|c|}{$(4,1)$} & \multicolumn{2}{|c|}{$(4,4)$} \\
\hline \hline \multirow{4}{*}{$\mathrm{N}=16$} & Fixtures & 1 & 2 & 3 & 4 & 5 & 6 & 7 & 8 \\
\cline { 2 - 10 } & Coordinates & $(1,1)$ & $(1,2)$ & $(1,3)$ & $(1,4)$ & $(2,1)$ & $(2,2)$ & $(2,3)$ & $(2,4)$ \\
\cline { 2 - 9 } & Fixtures & 9 & 10 & 11 & 12 & 13 & 14 & 15 & 16 \\
\cline { 2 - 9 } & Coordinates & $(3,1)$ & $(3,2)$ & $(3,3)$ & $(3,4)$ & $(4,1)$ & $(4,2)$ & $(4,3)$ & $(4,4)$ \\
\hline
\end{tabular}

performance between the BF search method and the proposed scheme. Moreover, it shows that the achievable secrecy rate is close to the secrecy capacity of the system. This results is consistent with results reported in [26] whereby it was shown that the optimal distribution that achieves the secrecy capacity of the MISO wiretap channel under an amplitude constraint is discrete with a finite support set. Moreover, it is can be seen from this figure that when Eve's location is not know, the use of AN enhance the secrecy performance of the system. In addition, we remark that the secrecy performance of the system increases as the number of LEDs fixtures at Alice increases or the average noise power decreases. This result is somehow expected, since both facts lead to increase the SNR of the receivers. On the other hand, note that the secrecy performance of the system depend on the amplitude constraint $A$. In constructing Fig. 3, we found that the best input distributions $p_{u}$ and $p_{x}$, for the information-bearing signal $u$ and for the jamming signal $x$, for the values of $A^{2}$ ranging from -40 $\mathrm{dBm}$ to $40 \mathrm{dBm}$ with a step size of $10 \mathrm{dBm}$. For example, for $N=16, A^{2}=0 \mathrm{dBm}$ and $\sigma^{2}=-98.82 \mathrm{dBm}$, we found numerically that the best probability distributions are $p_{u}=p_{x}=\mathrm{TDGN}(16, B, 0.5,2)$.

Another measure to evaluate the secrecy performance of the system is the modified secrecy outage probability (SOP). In our context, the modified SOP, denoted by $P_{\mathrm{SO}}$, is the probability that the he instantaneous achievable secrecy rate $R_{s}$, i.e., at a given location of Eve, is lower than a threshold secrecy rate $R_{t h}$, i.e

$$
P_{\text {SO }}=\mathbb{P}\left(R_{s} \leq R_{t h}\right) .
$$

For a fixed threshold secrecy rate, the secrecy performance of the system increases as the SOP decreases. The SOP was 


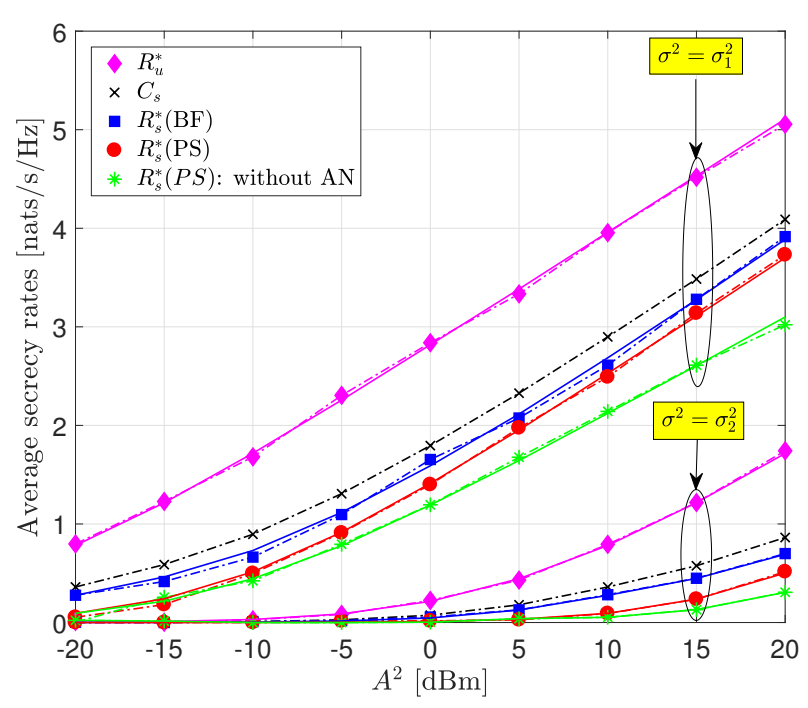

(a) $N=16$.

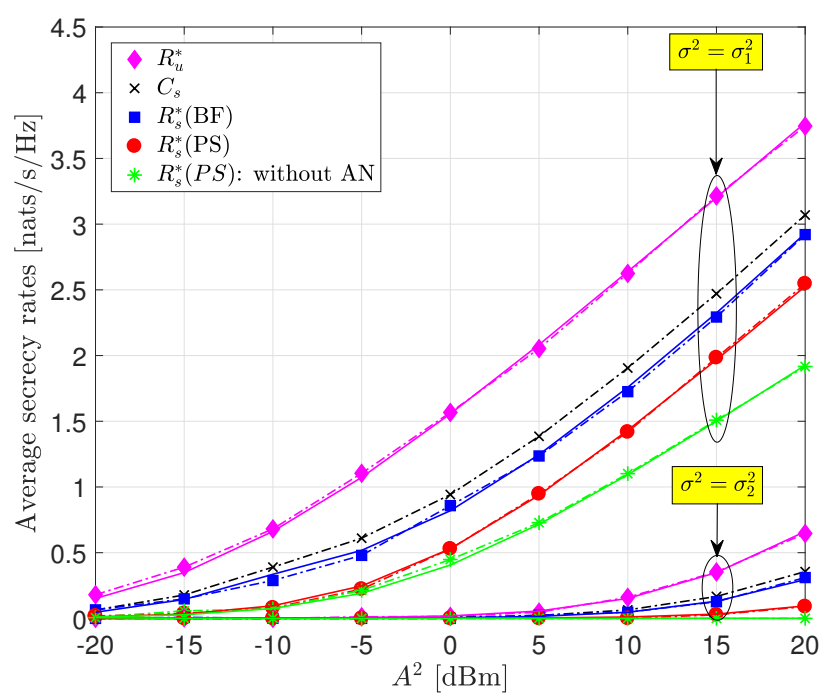

(b) $N=4$.

Fig. 3. Average upper bound $R_{u}^{*}$, average secrecy capacity $C_{s}$ and average achievable secrecy rates $R_{s}^{*}(B F)$ and $R_{s}^{*}(P S)$ versus $A^{2}$. Solid lines present theoretical results whereas dash-dotted lines present simulation results. $\sigma_{1}^{2}=$ $-98.82 \mathrm{dBm}$ and $\sigma_{2}^{2}=-68.82 \mathrm{dBm}$.

widely used in the literature, such as in [37], [38] for VLC systems. However, in [37], [38], the SOP was adopted for SISO VLC systems only due to the complex structure of the channel gain vector, when the transmitter is equipped with multiple fixtures of LEDs. Fig. 4, pretenses the empirical modified SOP versus the instantaneous achievable secrecy rate $R_{s}$ for the average noise powers $\sigma^{2}=-98.82 \mathrm{dBm}$ and $\sigma^{2}=-68.82 \mathrm{dBm}$ and for the numbers of Fixtures of LEDs at Alice $N=16$ and $N=4$. This figure shows also that increasing the number of fixtures of LEDs and/or decreasing the noise power at the receivers will decrease the secrecy outage probability of the system, and therefore, will increase the secrecy performance of the system.

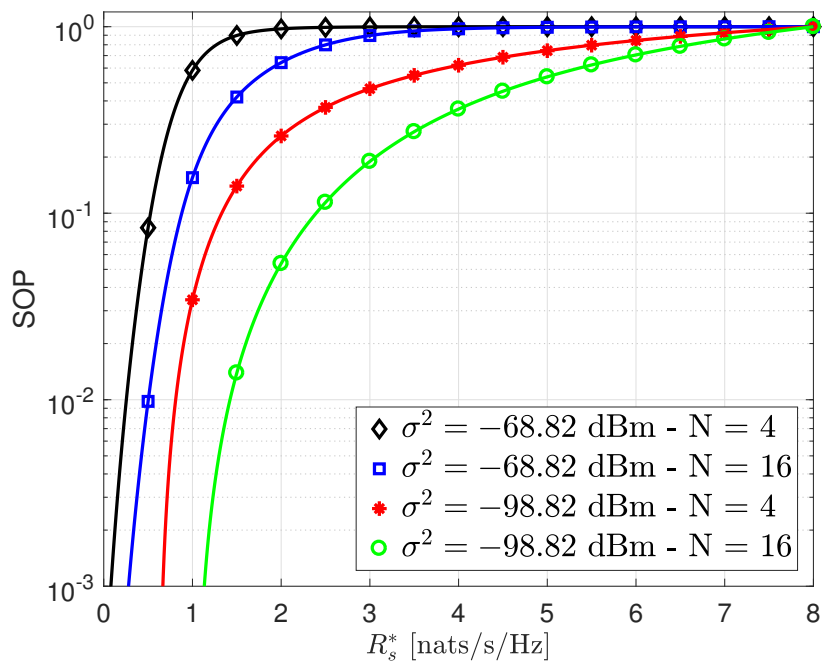

Fig. 4. Secrecy outage probability versus $R_{s}^{*}(P S)$ for various values of the noise variance $\sigma^{2}$ and different number of LEDs Fixtures $N$.

\section{CONClusion}

We studied in this paper the secrecy performance of the MISO VLC wiretap channel. We adopted AN-based beamforming as a transmission strategy in an effort to maximize the secrecy rate while degrading Eve's channel. We adopted discrete input signaling for both, the information bearing signal and the AN signal. Specifically, we derived achievable secrecy rates in closed-form expressions for the system as a function of the beamforming vector and the discrete input distribution. We investigated the problem of optimal beamfomring for the case imperfect Eve's CSI, where we assumed that Eve's location was unknown and only a coarse estimate of its CSI was available to Alice. Solving the optimization problem optimally was not possible. As such, we approached the solution in two different ways: numerically using $\mathrm{BF}$ methods and analytically using some approximations. The former is much more complex than the latter. To assess the performance of the proposed scheme, we employed the TDGN for the discrete input distribution where we optimized over its parameters. We corroborated the analytical results through Monte Carlo simulations and we demonstrated substantial improvements provided by the proposed scheme over existing ones. Furthermore, we showed that the approximations used in developing the proposed scheme result in marginal performance degradation as compared to that of the BF method (the optimal one.)

\section{APPENDIX A}

\section{PROOF OF THEOREM 1}

Based on the results of [25], a lower bound for the secrecy capacity of the system can be given by

$$
\begin{aligned}
C_{s} & \geq \max _{p_{\mathbf{u}}}\left[I\left(\mathbf{u} ; y_{B}\right)-I\left(\mathbf{u} ; y_{E}\right)\right]^{+} \\
& \geq\left[I\left(\mathbf{w}_{1} u ; y_{B}\right)-I\left(\mathbf{w}_{1} u ; y_{E}\right)\right]^{+} \\
& =h\left(y_{B}\right)-h\left(y_{B} \mid \mathbf{w}_{1} u\right)-h\left(y_{E}\right)+h\left(y_{E} \mid \mathbf{w}_{1} u\right)
\end{aligned}
$$


where inequality (50a) holds from [25] and inequality (50b) holds by letting $\mathbf{u}=\mathbf{w}_{1} u+\mathbf{w}_{2} x$ and choosing any variables $u$, $x, \mathbf{w}_{1}$ and $\mathbf{w}_{2}$. Now we develop each term of equation (50c). At first, we have

$$
h\left(y_{B} \mid \mathbf{w}_{1} u\right)=h\left(n_{B}\right)=\frac{1}{2} \log \left[2 \pi e \sigma_{B}^{2}\right] .
$$

Moreover, $h\left(y_{E}\right)=h\left(\mathbf{h}_{E}^{T} \mathbf{w}_{1} u+\mathbf{h}_{E}^{T} \mathbf{w}_{2} x+n_{E}\right)$ can be upper bounded by the differential entropy of a Gaussian distribution having the same variance, that is,

$$
\begin{aligned}
h\left(y_{E}\right) & \leq \frac{1}{2} \log \left[2 \pi e \operatorname{Var}\left(\mathbf{h}_{E}^{T} \mathbf{w}_{1} u+\mathbf{h}_{E}^{T} \mathbf{w}_{2} x+n_{E}\right)\right] \\
& =\frac{1}{2} \log \left[1+\rho_{E, u} \sigma_{u}^{2}+\rho_{E, x} \sigma_{x}^{2}\right]+\frac{1}{2} \log \left(2 \pi e \sigma_{E}^{2}\right) .
\end{aligned}
$$

Now we consider $h\left(y_{B} \mid \mathbf{w}_{1} u\right)$. We have $y_{B}=\mathbf{h}_{B}^{T} \mathbf{w}_{1} u+n_{B}$ and recall that the pmf of $u$ is given by

$$
p_{u}(z)=\sum_{i=1}^{K_{u}} p_{i} \delta\left(z-u_{i}\right) .
$$

In this case, $y_{B}$ is a mixture of Gaussian and its probability density function is given by

$$
p_{y_{B}}(y)=\sum_{i=1}^{K_{u}} \frac{p_{i}}{\sqrt{2 \pi \sigma_{B}^{2}}} \exp \left[-\frac{\left(y-\mathbf{h}_{B}^{T} \mathbf{w}_{1} u_{i}\right)^{2}}{2 \sigma_{B}^{2}}\right] .
$$

The derivation of a lower bound on the differential entropy of $y_{B}$ in this case is inspired from the one in [50]. Let $u^{c}$ be a Gaussian distribution that have the same variance as $u$, i.e., $u^{c}$ follows $\mathcal{N}\left(0, \sigma_{u}^{2}\right)$ and let $y_{B}^{c}=\mathbf{h}_{B}^{T} \mathbf{w}_{1} u^{c}+n_{B}$. In this case, $y_{B}^{c}$ follows a $\mathcal{N}\left(0, \sigma_{c}^{2}\right)$ distribution, where $\sigma_{c}^{2}=$ $\left(\mathbf{h}_{B}^{T} \mathbf{w}_{1}\right)^{2} \sigma_{u}^{2}+\sigma_{B}^{2}$. Since $y_{B}$ and $y_{B}^{c}$ have the same variance and using [51, Eq. 8.76], we have

$$
h\left(y_{B}^{c}\right)-h\left(y_{B}\right)=\mathcal{D}\left(y_{B} \| y_{B}^{c}\right)=\mathbb{E}_{y_{B}}\left(\log \left[\frac{p_{y_{B}}}{p_{y_{B}^{c}}}\right]\right),
$$

where $\mathcal{D}$ denotes Kullback-Leibler divergence. Moreover, using Jensen's inequality, we have

$$
\mathbb{E}_{y_{B}}\left(\log \left[\frac{p_{y_{B}}}{p_{y_{B}^{c}}}\right]\right) \leq \log \left[\mathbb{E}_{y_{B}}\left(\frac{p_{y_{B}}}{p_{y_{B}^{c}}}\right)\right] .
$$

Consequently, $h\left(y_{B}\right)$ is lower bounded as

$$
h\left(y_{B}\right) \geq \frac{1}{2} \log \left(2 \pi e \sigma_{c}^{2}\right)-\log \left[\mathbb{E}_{y_{B}}\left(\frac{p_{y_{B}}}{p_{y_{B}^{c}}}\right)\right],
$$

where $\mathbb{E}_{y_{B}}\left(\frac{p_{y_{B}}}{p_{y_{B}^{c}}^{c}}\right)$ is expressed as shown in equation (58) on top of next page, such that $a=\frac{2 \sigma_{c}^{2}-\sigma_{B}^{2}}{\sigma_{B}^{2} \sigma_{c}^{2}}$ and for all $(i, j) \in$ $\llbracket 1, K_{u} \rrbracket^{2}$,

$$
\left\{\begin{aligned}
b_{i, j} & =\frac{\sigma_{c}^{2}}{2 \sigma_{c}^{2}-\sigma_{B}^{2}} \mathbf{h}_{B}^{T} \mathbf{w}_{1}\left(u_{i}+u_{j}\right), \\
d_{i, j} & =\frac{-\rho_{B}}{4 \rho_{B}+2}\left(\rho_{B} \sigma_{u}^{2}\left(u_{i}-u_{j}\right)^{2}-2 u_{i} u_{j}\right) .
\end{aligned}\right.
$$

Consequently, since $\sigma_{c}^{2}=\left(\mathbf{h}_{B}^{T} \mathbf{w}_{1}\right)^{2} \sigma_{u}^{2}+\sigma_{B}^{2}$, we have $2 \sigma_{c}^{2}-$ $\sigma_{B}^{2}=2\left(\mathbf{h}_{B}^{T} \mathbf{w}_{1}\right)^{2} \sigma_{u}^{2}+\sigma_{B}^{2}$ and, therefore, we get

$$
\begin{aligned}
& \log \left[\mathbb{E}_{y_{B}}\left(\frac{p_{y_{B}}}{p_{y_{B}^{c}}}\right)\right]=\frac{1}{2} \log \left(2 \pi e \sigma_{c}^{2}\right)-\frac{1}{2} \log \left(2 \pi e \sigma_{B}^{2}\right) \\
& -\frac{1}{2}\left(\frac{1+2 \rho_{B} \sigma_{u}^{2}}{1+\rho_{B} \sigma_{u}^{2}}\right)-\log \left[\sum_{i=1}^{K_{u}} \sum_{j=1}^{K_{u}} p_{i} p_{j} \exp \left[d_{i, j}^{u}\right]\right] .
\end{aligned}
$$

Therefore, $h\left(y_{B}\right)$ is lower bounded as

$$
\begin{aligned}
h\left(y_{B}\right) & \geq \frac{1}{2} \log \left[\frac{1+2 \rho_{B} \sigma_{u}^{2}}{1+\rho_{B} \sigma_{u}^{2}}\right]-\log \left[\sum_{i=1}^{K_{u}} \sum_{j=1}^{K_{u}} p_{i} p_{j} \exp \left[d_{i, j}^{u}\right]\right] \\
& +\frac{1}{2} \log \left[2 \pi e \sigma_{B}^{2}\right] .
\end{aligned}
$$

For the last term, we have $h\left(y_{E} \mid \mathbf{w}_{1} u\right)=h\left(\mathbf{h}_{E}^{T} \mathbf{w}_{2} x+n_{E}\right)$, which, by using the same approach as for $h\left(y_{B}\right)$, can be lower bounded as

$$
\begin{aligned}
h\left(y_{E} \mid \mathbf{w}_{1} u\right) & \geq \frac{1}{2} \log \left[\frac{1+2 \rho_{E, x} \sigma_{x}^{2}}{1+\rho_{E, x} \sigma_{x}^{2}}\right]+\frac{1}{2} \log \left[2 \pi e \sigma_{E}^{2}\right] \\
& -\log \left[\sum_{i=1}^{K_{x}} \sum_{j=1}^{K_{x}} q_{i} q_{j} \exp \left[d_{i, j}^{x}\right]\right] .
\end{aligned}
$$

Finally, by substituting the above expressions in (50c), an achievable secrecy rate for the MISO VLC wiretap channel in (7) is given as shown in theorem 1, which completes the proof.

\section{APPENDIX B}

\section{PROOF OF LEMMA 1}

Consider the matrix $\mathbf{K}=d_{1} \mathbf{h}_{B} \mathbf{h}_{B}^{T}-d_{2} \mathbf{h}_{E} \mathbf{h}_{E}^{T}$ such that $d_{1}$, $d_{2} \in \mathbb{R}_{+}^{*}$ and $\mathbf{h}_{B}$ and $\mathbf{h}_{E}$ are not colinear. Our purpose in this section is to characterize the spectrum of the matrix $\mathbf{K}$. The matrix $\mathbf{K}$ is symmetric and, thus, it is diagonalizable. Moreover, since $\mathbf{h}_{B}$ and $\mathbf{h}_{E}$ are not colinear, the rank of $\mathbf{K}$ is equal to two. Therefore, $\mathbf{K}$ have exactly two non-zero eigenvalues. Let $\lambda \in \mathbb{R}$ be a non-zero eigenvalue of $\mathbf{K}$ and e be its associated eigenvector. Motivated by the structure of $\mathbf{K}$, we suppose that there exists $\mathbf{a}_{1}, \mathbf{a}_{2} \in \mathbb{R}$ such that $\mathbf{e}=a_{1} \mathbf{h}_{B}+a_{2} \mathbf{h}_{E}$. Consequently, we get

$$
\begin{aligned}
\mathbf{K e} & =d_{1}\left(a_{1}\left\|\mathbf{h}_{B}\right\|_{2}^{2}+a_{2} \mathbf{h}_{B}^{T} \mathbf{h}_{E}\right) \mathbf{h}_{B} \\
& -d_{2}\left(a_{1} \mathbf{h}_{B}^{T} \mathbf{h}_{E}+a_{2}\left\|\mathbf{h}_{E}\right\|_{2}^{2}\right) \mathbf{h}_{E} .
\end{aligned}
$$

In addition, we have $\mathbf{K e}=\lambda \mathbf{e}=\lambda a_{1} \mathbf{h}_{B}+\lambda a_{2} \mathbf{h}_{E}$. Based on this, one way of equality between $\mathbf{K e}$ and $\lambda \mathbf{e}$, is setting

$$
\left\{\begin{aligned}
d_{1}\left(a_{1}\left\|\mathbf{h}_{B}\right\|_{2}^{2}+a_{2} \mathbf{h}_{B}^{T} \mathbf{h}_{E}\right) & =\lambda a_{1} \\
-d_{2}\left(a_{1} \mathbf{h}_{B}^{T} \mathbf{h}_{E}+a_{2}\left\|\mathbf{h}_{E}\right\|_{2}^{2}\right) & =\lambda a_{2} .
\end{aligned}\right.
$$

If $\mathbf{h}_{B}^{T} \mathbf{h}_{E}=0$, in this case, $\mathbf{K}$ have one positive eigenvalue $\lambda_{1}=d_{1}\left\|\mathbf{h}_{B}\right\|_{2}$ associated to the eigenvector $\mathbf{e}_{1}=\frac{\mathbf{h}_{B}}{\left\|\mathbf{h}_{B}\right\|_{2}}$ and one negative eigenvalue $\lambda_{2}=-d_{2}|| \mathbf{h}_{E} \|_{2}$ associated to the 


$$
\begin{aligned}
\mathbb{E}_{y_{B}}\left(\frac{p_{y_{B}}}{p_{y_{B}^{c}}}\right) & =\int_{-\infty}^{\infty} p_{y_{B}}(y) \frac{p_{y_{B}}(y)}{p_{y_{B}^{c}}(y)} \mathrm{d} y \\
& =\sum_{i=1}^{K_{u}} \sum_{j=1}^{K_{u}} \frac{\left(2 \pi \sigma_{c}^{2}\right)^{\frac{1}{2}} p_{i} p_{j}}{2 \pi \sigma_{B}^{2}} \int_{-\infty}^{\infty} \exp \left[-\frac{1}{2}\left(\frac{\left(y-\mathbf{h}_{B}^{T} \mathbf{w}_{1} u_{i}\right)^{2}}{\sigma_{B}^{2}}+\frac{\left(y-\mathbf{h}_{B}^{T} \mathbf{w}_{1} u_{j}\right)^{2}}{\sigma_{B}^{2}}-\frac{y^{2}}{\sigma_{c}^{2}}\right)\right] \mathrm{d} y \\
& =\frac{\left(2 \pi \sigma_{c}^{2}\right)^{\frac{1}{2}}}{2 \pi \sigma_{B}^{2}} \sum_{i=1}^{K_{u}} \sum_{j=1}^{K_{u}} p_{i} p_{j} \int_{-\infty}^{\infty} \exp \left[-\frac{1}{2}\left(\left(\frac{2}{\sigma_{B}^{2}}-\frac{1}{\sigma_{c}^{2}}\right) y^{2}-\frac{2 \mathbf{h}_{B}^{T} \mathbf{w}_{1}}{\sigma_{B}^{2}}\left(u_{i}+u_{j}\right) y+\frac{\left(\mathbf{h}_{B}^{T} \mathbf{w}_{1}\right)^{2}}{\sigma_{B}^{2}}\left(u_{i}^{2}+u_{j}^{2}\right)\right)\right] \mathrm{d} y \\
& =\frac{\left(2 \pi \sigma_{c}^{2}\right)^{\frac{1}{2}}}{2 \pi \sigma_{B}^{2}} \sum_{i=1}^{K_{u}} \sum_{j=1}^{K_{u}} p_{i} p_{j} \int_{-\infty}^{\infty} \exp \left[-\frac{a}{2}\left(y-b_{i, j}\right)^{2}+d_{i, j}^{u}\right] \mathrm{d} y \\
& =\frac{\left(2 \pi \sigma_{c}^{2}\right)^{\frac{1}{2}}}{2 \pi \sigma_{B}^{2}} \sum_{i=1}^{K_{u}} \sum_{j=1}^{K_{u}} p_{i} p_{j} \exp \left[d_{i, j}^{u}\right]\left(\int_{-\infty}^{\infty} \exp \left[-\frac{a}{2}\left(y-b_{i, j}\right)^{2}\right] \mathrm{d} y\right) \\
& =\frac{\left(2 \pi \sigma_{c}^{2}\right)^{\frac{1}{2}}}{2 \pi \sigma_{B}^{2}}\left(\frac{2 \pi \sigma_{B}^{2} \sigma_{c}^{2}}{2 \sigma_{c}^{2}-\sigma_{B}^{2}}\right)^{\frac{1}{2}} \sum_{i=1}^{K_{u}} \sum_{j=1}^{K_{u}} p_{i} p_{j} \exp \left[d_{i, j}^{u}\right] \\
& =\frac{\left(2 \pi e \sigma_{c}^{2}\right)^{\frac{1}{2}}}{\left(2 \pi e \sigma_{B}^{2}\right)^{\frac{1}{2}}}\left(\frac{\sigma_{c}^{2}}{2 \sigma_{c}^{2}-\sigma_{B}^{2}}\right)^{\frac{1}{2}} \sum_{i=1}^{K_{u}} \sum_{j=1}^{K_{u}} p_{i} p_{j} \exp \left[d_{i, j}^{u}\right] .
\end{aligned}
$$

eigenvector $\mathbf{e}_{2}=\frac{\mathbf{h}_{E}}{\left\|\mathbf{h}_{E}\right\|_{2}}$.On the other hand, if $\mathbf{h}_{B}^{T} \mathbf{h}_{E}^{T} \neq 0$, then the system in (64) is equivalent to

$$
\left[\begin{array}{cc}
d_{1}\left\|\mathbf{h}_{B}\right\|_{2}^{2} & d_{1} \mathbf{h}_{B}^{T} \mathbf{h}_{E} \\
-d_{2} \mathbf{h}_{B}^{T} \mathbf{h}_{E} & -d_{2}\left\|\mathbf{h}_{E}\right\|_{2}^{2}
\end{array}\right]\left[\begin{array}{c}
a_{1} \\
a_{2}
\end{array}\right]=\lambda\left[\begin{array}{c}
a_{1} \\
a_{2}
\end{array}\right] .
$$

Based on (65), the eigenvalues of $\mathbf{K}$ are the roots of the characteristic polynomial of the system matrix, i.e., solutions of the equation

$$
\begin{aligned}
(E): \quad \lambda^{2} & -\left(d_{1}\left\|\mathbf{h}_{B}\right\|_{2}^{2}-d_{2}\left\|\mathbf{h}_{E}\right\|_{2}^{2}\right) \lambda \\
& -d_{1} d_{2}\left(\left\|\mathbf{h}_{B}\right\|_{2}^{2}\left\|\mathbf{h}_{E}\right\|_{2}^{2}-\left(\mathbf{h}_{B}^{T} \mathbf{h}_{E}\right)^{2}\right)=0 .
\end{aligned}
$$

The discriminant of equation $(E)$ is

$$
\begin{aligned}
\Delta= & \left(d_{1}\left\|\mathbf{h}_{B}\right\|_{2}^{2}-d_{2}\left\|\mathbf{h}_{E}\right\|_{2}^{2}\right)^{2} \\
& +4 d_{1} d_{2}\left(\left\|\mathbf{h}_{B}\right\|_{2}^{2}\left\|\mathbf{h}_{E}\right\|_{2}^{2}-\left(\mathbf{h}_{B}^{T} \mathbf{h}_{E}\right)^{2}\right)
\end{aligned}
$$

which is always positive due to the inequality of Cauchy Schwarz. Consequently, equation $(E)$ has two roots which are

$$
\left\{\begin{array}{l}
\lambda_{1}=\frac{d_{1}\left\|\mathbf{h}_{B}\right\|_{2}^{2}-d_{2}|| \mathbf{h}_{E} \|_{2}^{2}}{2}+\frac{\sqrt{\Delta}}{2} \\
\lambda_{2}=\frac{d_{1}\left\|\mathbf{h}_{B}\right\|_{2}^{2}-d_{2}\left\|\mathbf{h}_{E}\right\|_{2}^{2}}{2}-\frac{\sqrt{\Delta}}{2} .
\end{array}\right.
$$

Moreover, since $d_{1}\left\|\mathbf{h}_{B}\right\|_{2}^{2}-d_{2}\left\|\mathbf{h}_{E}\right\|_{2}^{2} \leq \Delta$, we have $\lambda_{1} \geq 0$ and $\lambda_{2} \leq 0$. Finally, for each eigenvalue $\lambda=\left\{\lambda_{1}, \lambda_{2}\right\}$, we inject $\lambda$ in the system in (64) and we solve. Consequently, for each eigenvalue $\lambda=\left\{\lambda_{1}, \lambda_{2}\right\}$, the associated eigenvector of $\lambda$ is given by $\mathbf{e}\left(\mathbf{h}_{E}\right)=a_{1} \mathbf{h}_{B}+a_{2} \mathbf{h}_{E}$, where

$$
\left\{\begin{array}{l}
a_{1}=\frac{\left(\lambda+d_{2}|| \mathbf{h}_{E} \|_{2}^{2}\right)\left\|\mathbf{h}_{B}\right\|_{2}^{2}-d_{1}\left(\mathbf{h}_{B}^{T} \mathbf{h}_{E}\right)^{2}-\lambda^{2}}{\lambda d_{2}\left\|\mathbf{h}_{E}\right\|_{2}^{2}}, \\
a_{2}=\frac{-d_{1}\left(\mathbf{h}_{B}^{T} \mathbf{h}_{E}\right)}{\lambda+d_{2}|| \mathbf{h}_{E} \|_{2}^{2}} a_{1} .
\end{array}\right.
$$

After that, we can ensure that each eigenvector is normal by dividing each coefficient in (69) by $\sqrt{a_{1}^{2}+a_{2}^{2}}$.

\section{REFERENCES}

[1] A. R. Ndjiongue, H. C. Ferreira, and T. Ngatched, "Visible light communications (VLC) technology," Wiley Encyclopedia of Electrical and Electronics Engineering, Jun. 2015.

[2] S. Rajagopal, R. D. Roberts, and S.-K. Lim, "IEEE 802.15. 7 visible light communication: modulation schemes and dimming support," IEEE Commun Mag., vol. 50, no. 3, pp. 72-82, Mar. 2012.

[3] S. Gao, "Performance study for indoor visible light communication systems," Ph.D. dissertation, University of Ottawa, 2013.

[4] D. K. Borah, A. C. Boucouvalas, C. C. Davis, S. Hranilovic, and K. Yiannopoulos, "A review of communication-oriented optical wireless systems," EURASIP J. Wireless Commun. and Net., vol. 2012, no. 1, pp. 1-28, Mar. 2012.

[5] M. A. Khalighi and M. Uysal, "Survey on free space optical communication: A communication theory perspective," IEEE Commun. Surveys \& Tutorials, vol. 16, no. 4, pp. 2231-2258, Jun. 2014.

[6] D. Karunatilaka, F. Zafar, V. Kalavally, and R. Parthiban, "LED based indoor visible light communications: State of the art," IEEE Commun. Surveys \& Tutorials, vol. 17, no. 3, pp. 1649-1678, Mar. 2015.

[7] H. Elgala, R. Mesleh, and H. Haas, "Indoor optical wireless communication: potential and state-of-the-art," IEEE Commun. Mag., vol. 49, no. 9, pp. 56-62, Sep. 2011.

[8] A. Chaaban, Z. Rezki, and M.-S. Alouini, "Fundamental limits of parallel optical wireless channels: Capacity results and outage formulation," IEEE Trans. Commun., vol. 65, no. 1, pp. 296-311, Oct. 2016. 
[9] A. Lapidoth, S. M. Moser, and M. A. Wigger, "On the capacity of freespace optical intensity channels," IEEE Trans. Inform. Theory, vol. 55, no. 10, pp. 4449-4461, Sep. 2009.

[10] A. A. Farid and S. Hranilovic, "Capacity bounds for wireless optical intensity channels with Gaussian noise," IEEE Trans. Inform. Theory, vol. 56, no. 12, pp. 6066-6077, Nov. 2010.

[11] S. Wu, H. Wang, and C.-H. Youn, "Visible light communications for 5G wireless networking systems: from fixed to mobile communications," IEEE Network, vol. 28, no. 6, pp. 41-45, Nov. 2014.

[12] J. Classen, J. Chen, D. Steinmetzer, M. Hollick, and E. Knightly, "The spy next door: Eavesdropping on high throughput visible light communications," in Proc. ACM, Paris, France, Sep. 2015.

[13] A. Yener and S. Ulukus, "Wireless physical-layer security: lessons learned from information theory," Proc. IEEE, vol. 103, no. 10, pp. 1814-1825, Sep. 2015.

[14] T. Bilski, "New threats and innovative protection methods in wireless transmission systems," J. of Telecommun. and Inform. Technology, vol. 3, pp. 26-33, Mar. 2014.

[15] Y.-S. Shiu, S. Y. Chang, H.-C. Wu, S. C.-H. Huang, and H.-H. Chen, "Physical layer security in wireless networks: A tutorial," IEEE Trans. Wireless Commun., vol. 18, no. 2, pp. 66-74, Apr. 2011.

[16] H. Elgala, R. Mesleh, and H. Haas, "An led model for intensitymodulated optical communication systems," IEEE Photonics Tech. Letters, vol. 22, no. 11, pp. 835-837, Apr. 2010.

[17] Z. Li, W. Trappe, and R. Yates, "Secret communication via multi-antenna transmission," in Proc. IEEE CISS, MD, USA, Sep. 2007.

[18] S. Shafiee and S. Ulukus, "Achievable rates in gaussian MISO channels with secrecy constraints," in Proc. IEEE ISIT, Nice, France, Jun. 2007.

[19] J. Li and A. Petropulu, "Optimal input covariance for achieving secrecy capacity in Gaussian MIMO wiretap channels," in Proc. IEEE ICASSP., TX, USA, Mar. 2010.

[20] A. Khisti and G. W. Wornell, "Secure transmission with multiple antennas I: The MISOME wiretap channel," IEEE Trans. Inform. Theory, vol. 56, no. 7, pp. 3088-3104, Jun. 2010.

[21] W. Shi and J. Ritcey, "Robust beamforming for MISO wiretap channel by optimizing the worst-case secrecy capacity," in Proc. IEEE Asilomar, CA, USA, Apr. 2011.

[22] Q. Li and W.-K. Ma, "Optimal and robust transmit designs for MISO channel secrecy by semidefinite programming," IEEE Trans. on Signal Process., vol. 59, no. 8, pp. 3799-3812, Apr. 2011.

[23] G. Chen, Y. Gong, P. Xiao, and J. A. Chambers, "Physical layer network security in the full-duplex relay system," IEEE Trans. Inform. forensics and security, vol. 10, no. 3, pp. 574-583, Jan. 2015.

[24] G. Chen, Y. Gong, P. Xiao, and J. A. Chambers, "Dual antenna selection in secure cognitive radio networks," IEEE trans. Vehicular Tech., vol. 65, no. 10, pp. 7993-8002, Dec. 2016.

[25] I. Csiszár and J. Korner, "Broadcast channels with confidential messages," IEEE Trans. Inform. Theory, vol. 24, no. 3, pp. 339-348, May. 1978.

[26] Z. Rezki and M.-S. Alouini, "Secret-key agreement with public discussion over multi-antenna aransmitters with amplitude constraints," in Proc. IEEE ISIT, Aachen, Germany, Aug. 2017, pp. 1534-1538.

[27] A. Mostafa and L. Lampe, "Physical-layer security for MISO visible light communication channels," IEEE J. on Selected Areas in Commun., vol. 33, no. 9, pp. 1806-1818, May 2015.

[28] A. Mostafa and L. Lampe, "Optimal and robust beamforming for secure transmission in MISO visible-light communication links," IEEE Trans. Signal Process., vol. 64, no. 24, pp. 6501-6516, Aug. 2016.

[29] M.-A. Arfaoui, Z. Rezki, A. Ghrayeb, and M. S. Alouini, "On the input distribution and optimal beamforming for the MISO VLC wiretap channel," in Proc. IEEE GlobalSIP, Washington DC, USA, Dec. 2016.

[30] H. Zaid, Z. Rezki, A. Chaaban, and M. S. Alouini, "Improved secrecy rate of visible light communication with cooperative jamming," in Proc. IEEE GlobalSIP, FL, USA, Dec. 2015.

[31] M.-A. Arfaoui, Z. Rezki, A. Ghrayeb, and M. S. Alouini, "On the secrecy capacity of MISO visible light communication channels," in Proc. IEEE Globecom, Washington DC, USA, Dec. 2016.

[32] A. Mostafa and L. Lampe, "Securing visible light communications via friendly jamming," in Proc. IEEE Globecom Workshops, Austin, TX, USA, Dec. 2014.

[33] H. Shen, Y. Deng, W. Xu, and C. Zhao, "Secrecy-oriented transmitter optimization for visible light communication systems," IEEE Photonics Journal, vol. 8, no. 5, pp. 1-14, Aug. 2016.

[34] D. Zou, C. Gong, and Z. Xu, "Secrecy rate of MISO optical wireless scattering communications," IEEE Trans. Commun., vol. 66, no. 1, pp. 225-238, Jul. 2017.
[35] F. Wang, C. Liu, Q. Wang, J. Zhang, R. Zhang, L.-L. Yang, and L. Hanzo, "Optical jamming enhances the secrecy performance of the generalized space shift keying aided visible light downlink," IEEE Trans. Commun., Apr. 2018.

[36] G. Chen, J. P. Coon, and M. Di Renzo, "Secrecy outage analysis for downlink transmissions in the presence of randomly located eavesdroppers," IEEE Trans. Inform. Forensics and Security, vol. 12, no. 5, pp. 1195-1206, Jan. 2017.

[37] G. Pan, J. Ye, and Z. Ding, "On secure VLC systems with spatially random terminals," IEEE Commun. Letters, vol. 21, no. 3, pp. 492-495, Dec. 2017.

[38] S. Cho, G. Chen, and J. P. Coon, "Physical layer security in visible light communication systems with randomly located colluding eavesdroppers," IEEE Wireless Commun. Letters, vol. 7, no. 5, pp. $768-$ 771, Oct. 2018.

[39] S. Cho, G. Chen, and J. P. Coon, "Securing visible light communication systems by beamforming in the presence of randomly distributed eavesdroppers," IEEE Trans. Wireless Commun., vol. 17, no. 5, pp. 29182931, Feb. 2018.

[40] S. Cho, G. Chen, and J. P. Coon, "Secrecy analysis in visible light communication systems with randomly located eavesdroppers," in Proc. IEEE ICC, Paris, France, May. 2017.

[41] J. Kahn and J. Barry, "Wireless infrared communications," Proc. of the IEEE, vol. 85, no. 2, pp. 265-298, Feb. 1997.

[42] L. Zeng, D. C. O'Brien, H. Le Minh, G. E. Faulkner, K. Lee, D. Jung, Y. Oh, and E. T. Won, "High data rate multiple input multiple output (MIMO) optical wireless communications using white LED lighting," IEEE J. Select. Areas in Commun., vol. 27, no. 9, pp. 1654 - 1662, Dec. 2009.

[43] V. W. Chan, "Free-space optical communications," J. of Lightwave Tech., vol. 24, no. 12, pp. 4750-4762, Dec. 2006.

[44] O. Ozel, E. Ekrem, and S. Ulukus, "Gaussian wiretap channel with amplitude and variance constraints," IEEE Trans. Inform. Theory, vol. 61, no. 10 , pp. 5553 - 5563, Jul. 2015.

[45] T. Lipp and S. Boyd, "Variations and extension of the convex-concave procedure," Optimization and Engineering, vol. 17, no. 2, pp. 263-287, Nov. 2015.

[46] M. Grant, S. Boyd, and Y. Ye, "Cvx: Matlab software for disciplined convex programming," Web page and software available at http://cvxr. com/cvx/, Dec. 2017.

[47] J. Lofberg, "Yalmip: A toolbox for modeling and optimization in matlab," in Proc IEEE ICRA, New Orleans, LA, USA, Sep. 2004.

[48] Y. Nesterov and A. Nemirovskii, Interior-point polynomial algorithms in convex programming. Siam, 1994, vol. 13.

[49] S. Loyka and C. D. Charalambous, "Optimal signaling for secure communications over gaussian MIMO wiretap channels," IEEE Trans. Inform. Theory, vol. 62, no. 12, pp. 7207-7215, Sep. 2016.

[50] M. U. Baig, A. Nosratinia, and A. Host-Madsen, "Discrete modulation for interference mitigation," in Proc. IEEE ISIT, Barcelona, Spain, Aug. 2017.

[51] T. M. Cover and J. A. Thomas, Elements of Information Theory 2nd Edition. Wiley-Interscience, 2006.

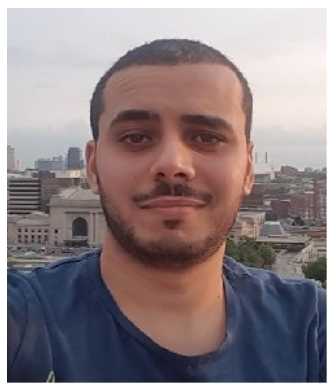

Mohamed Amine Arfaoui received the B.E. degree in electrical and computer engineering from the École Polytechnique de Tunisie, Tunisia, in 2015, and the M.Sc. degree in information systems engineering from Concordia University, Montreal, QC, Canada, in 2017. He is currently pursuing the Ph.D. degree in information systems engineering with Concordia University, Montreal. His current research interests include communication theory, optical communications and physical layer security. 


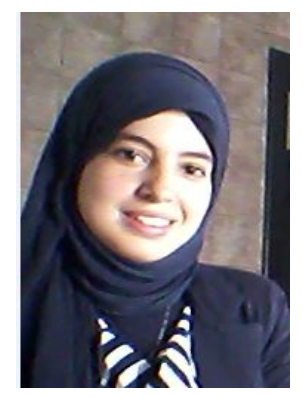

Hajar Zaid Received her Degree as a state engineer in network and telecommunication from the national school of applied sciences in OujdaMorocco 2015; the defense thesis was entitled Security of visible light communication with cooperative jamming. Certified CCNA Routing and Switching and currently works as a network and security engineer in a multinational company in Casablanca Morocco.

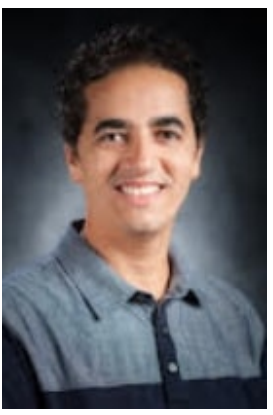

Zouheir Rezki was born in Casablanca, Morocco. He received the Diplome d'Ingenieur degree from the Ecole Nationale de l'Industrie Minerale (ENIM), Rabat, Morocco, in 1994, the M.Eng. degree from Ecole de Technologie Superieure, Montreal, Quebec, Canada, in 2003, and the Ph.D. degree in electrical engineering from Ecole Polytechnique, Montreal, Quebec, in 2008. After a few years of experience as a postdoctoral fellow and a research scientist at KAUST, he joined University of Idaho as an Assistant Professor in the ECE Department. .

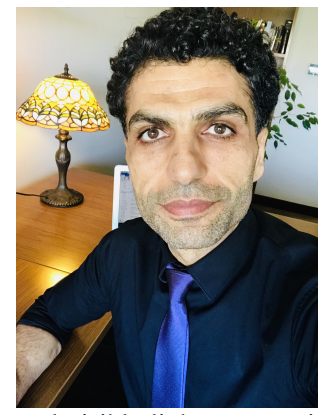

Ali Ghrayeb received the Ph.D. degree in electrical engineering from The University of Arizona, Tucson, AZ, USA, in 2000 . He is currently a Professor with the Department of Electrical and Computer Engineering, Texas A\&M University at Qatar. Prior to his current position, he was a professor with the Department of Electrical and Computer Engineering, Concordia University, Montreal, Canada. He has co-authored two books and published over 200 journal and conference papers. His research interests include wireless and mobile communications, physical layer security, massive MIMO, and visible light communications. He served as an instructor or co-instructor in technical tutorials at several major IEEE conferences. He served as the Executive Chair for the 2016 IEEE WCNC conference. He has served on the editorial board of several IEEE and non-IEEE journals. He is a Fellow of the IEEE..

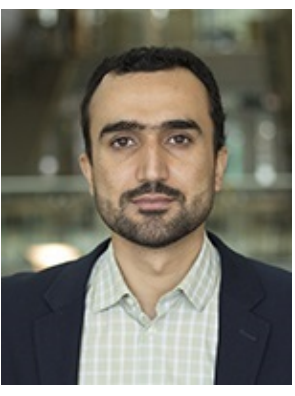

Anas Chaaban received the Maitrise ès Sciences degree in electronics from Lebanese University, Lebanon, in 2006, the M.Sc. degree in communications technology and the Dr. Ing. (Ph.D.) degree in electrical engineering and information technology from the University of Ulm and the Ruhr University of Bochum, Germany, in 2009 and 2013, respectively. From 2008 to 2009, he was with the Daimler AG Research Group On Machine Vision, Ulm, Germany. He was a Research Assistant with the Emmy-Noether Research Group on Wireless Networks, University of Ulm, Germany, from 2009 to 2011, which relocated to the Ruhr-University of Bochum in 2011. He was a PostDoctoral Researcher with the Ruhr-University of Bochum from 2013 to 2014, and with King Abdullah University of Science and Technology from 2015 to 2017. He joined the School of Engineering at the University of British Columbia as an Assistant Professor in 2018. His research interests are in the areas of information theory and wireless communications.

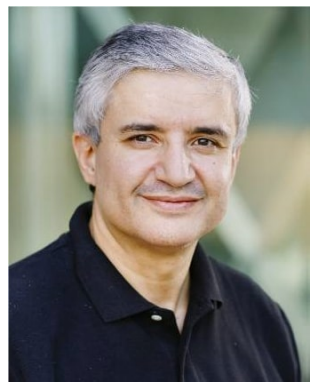

Mohamed-Slim Alouini was born in Tunis, Tunisia. He received the Ph.D. degree in Electrical Engineering from the California Institute of Technology (Caltech), Pasadena, CA, USA, in 1998. He served as a faculty member in the University of Minnesota, Minneapolis, MN, USA, then in the Texas A\&M University at Qatar, Education City, Doha, Qatar before joining King Abdullah University of Science and Technology (KAUST), Thuwal, Makkah Province, Saudi Arabia as a Professor of Electrical Engineering in 2009. His current research interests include of wireless communication systems. the modeling, design, and performance analysis 